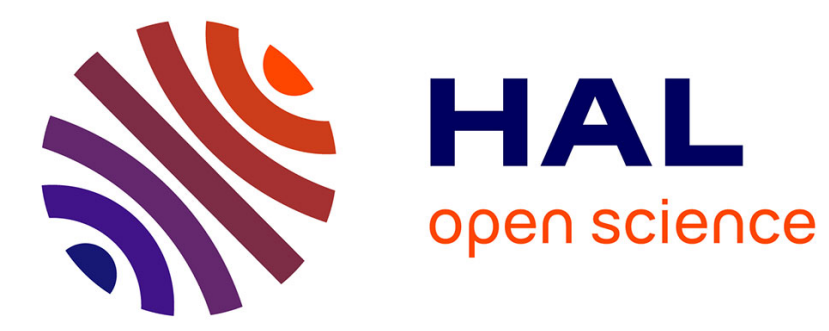

\title{
Topology-preserving rigid transformation of 2D digital images
}

Phuc Ngo, Nicolas Passat, Yukiko Kenmochi, Hugues Talbot

\section{To cite this version:}

Phuc Ngo, Nicolas Passat, Yukiko Kenmochi, Hugues Talbot. Topology-preserving rigid transformation of 2D digital images. IEEE Transactions on Image Processing, 2014, 23 (2), pp.885 - 897. 10.1109/TIP.2013.2295751 . hal-00795054v1

\section{HAL Id: hal-00795054 https://hal.science/hal-00795054v1}

Submitted on 3 Jul 2013 (v1), last revised 22 Apr 2014 (v2)

HAL is a multi-disciplinary open access archive for the deposit and dissemination of scientific research documents, whether they are published or not. The documents may come from teaching and research institutions in France or abroad, or from public or private research centers.
L'archive ouverte pluridisciplinaire HAL, est destinée au dépôt et à la diffusion de documents scientifiques de niveau recherche, publiés ou non, émanant des établissements d'enseignement et de recherche français ou étrangers, des laboratoires publics ou privés. 


\title{
Topology-preserving rigid transformation of $2 \mathrm{D}$ digital images
}

\author{
Phuc Ngo, Nicolas Passat, Yukiko Kenmochi, Hugues Talbot
}

\begin{abstract}
We provide conditions under which 2D digital images, considered in the two most common digital topology models (namely, dual adjacency and well-composedness), preserve their topological properties under rigid transformation. This study, that is developed in a discrete framework, leads to the proposal of efficient preprocessing strategies that ensure the topological invariance of images under further rigid transformation. These results and methods are proved to be valid for various kinds of images (binary, grey-level, label), thus providing a generic set of tools, that can be used in particular in the context of image registration and warping.
\end{abstract}

Index Terms-Digital imaging, rigid transformation, digital topology, well-composed images, image correction.

\section{INTRODUCTION}

$\mathbf{I}_{\mathrm{i}}^{\mathrm{s}}$ $\mathrm{N}$ digital imaging, the preservation of topological properties is a crucial issue in several application fields, involving 3D data (e.g., medical imaging [1]) but also 2D ones (e.g., remote sensing [2]). In particular, topology preservation -pioneered nearly fifty years ago [3], [4]- has been investigated in the context of image transformation, both from the viewpoints of registration [5] and warping [6]. It has to be noticed that efforts have been mainly devoted to handle complex transformations, while more simple ones have been globally unconsidered.

Indeed, the handling of "simple" transformations (e.g., translations, rotations) is often assumed to be trivial. This can be explained by the fact that, in the continuous case (i.e., in $\mathbb{R}^{n}$ ), most of such transformations are topology-preserving, while this is not necessarily the case for complex ones (e.g., those induced by nonrigid registration [7]). Based on this "continuous" assertion, it is often thought that simple transformations still lead to easy handling of topological properties in the digital case (i.e., in $\mathbb{Z}^{n}$ ). This is a wrong belief.

In the case of rigid transformations [8], that include the family of rotations, (e.g., (quasi-)shear rotations [9], [10], or hinge angle rotations [11], [12], [13]), some topological issues have been identified [14], [15]. These issues are directly or indirectly induced by the sampling policies that are mandatory to guarantee the stability of the transformations inside $\mathbb{Z}^{n}$.

In this article, we propose a study devoted to the topological invariance of 2D digital images under rigid transformation. In Sec. II, we first provide background notions required to make the article self-contained. Secs. III-V constitute the core of the article. The main purposes are first detailed in Sec. III.

Phuc Ngo, Yukiko Kenmochi and Hugues Talbot are with the ESIEEParis and the Université Paris-Est, LIGM UMR CNRS 8049, Paris, France ( $\{$ p.ngo,y.kenmochi,h.talbot $\} @$ esiee.fr).

Nicolas Passat is with the Université de Reims Champagne-Ardenne, CReSTIC EA 3804, Reims, France (nicolas.passat@univ-reims.fr).
We then propose, in Sec. IV, some conditions under which a 2D digital image preserves its topological properties under any rigid transformation. Based on these results, we provide in Sec. V some methodological solutions for analysing and preprocessing digital images before rigid transformation, in order to preserve their topological properties. This study is generic on two sides: $(i)$ the main two digital topology models are considered, namely the dual adjacency, and the wellcomposedness ones; and $(i i)$ the cases of binary, grey-level and label images are dealt with. Sec. VI concludes the article by perspective works. For the sake of readability, technical proofs are reported in Appendix.

\section{BACKGROUND NOTIONS}

\section{A. Notations}

The sets are noted $\mathbb{A}, \mathbb{B}, \mathbb{C}$, etc. Subsets of these sets are noted $\mathrm{A}, \mathrm{B}, \Gamma$, etc. The power set of a set $\mathrm{A}$ is noted $2^{\mathrm{A}}$. The elements of sets are noted $a, b, c$, etc., and $\mathbf{a}, \mathbf{b}, \mathbf{c}$, etc. if the set is a cartesian product. By abuse of notation, an element a, that should be noted as a column vector, is noted as a line vector, e.g., $\mathbf{a}=(a, b)$ instead of $\mathbf{a}=\left(\begin{array}{l}a \\ b\end{array}\right)$.

The functions defined on continuous sets are noted $\mathcal{A}, \mathcal{B}, \mathcal{C}$, etc., and the ones defined on discrete sets are noted $A, B, C$, etc. A function $F$ from $\mathbb{A}$ to $\mathbb{B}$ is noted $F: \mathbb{A} \rightarrow \mathbb{B}$. If $\mathrm{A} \subseteq \mathbb{A}$ and $\mathrm{B} \subseteq \mathbb{B}$, we note $F(\mathrm{~A})=\{F(x) \mid x \in \mathrm{A}\}$ and $F^{-1}(\mathrm{~B})=$ $\{x \mid F(x) \in \mathrm{B}\}$. If $F$ is a bijection, its inverse function is also noted $F^{-1}: \mathbb{B} \rightarrow \mathbb{A}$. The restriction of $F: \mathbb{A} \rightarrow \mathbb{B}$ to the subset $\mathrm{A} \subseteq \mathbb{A}$ is noted $F_{\mid \mathrm{A}}: \mathrm{A} \rightarrow \mathbb{B}$. The composition of $F: \mathbb{A} \rightarrow \mathbb{B}$ and $G: \mathbb{B} \rightarrow \mathbb{C}$ is noted $G \circ F: \mathbb{A} \rightarrow \mathbb{C}$. The spaces of functions are noted $\mathfrak{A}, \mathfrak{B}, \mathfrak{C}$, etc.

Adjacency (i.e., binary, irreflexive and symmetric) relations are noted $\frown$. Equivalence (i.e., binary, reflexive, transitive and symmetric) relations are noted $\sim$. We recall that a relation $\frown$ (resp. $\sim$ ) defined on a set $\mathrm{A}$ is actually a subset of $\mathrm{A} \times \mathrm{A}$, and that $a \frown b$ (resp. $a \sim b)$ means that $(a, b) \in \frown($ resp. $\sim$ ). Given a set $\mathrm{A}$, equipped with an equivalence relation $\sim$, the equivalence class of $a \in \mathrm{A}$ with respect to $\sim$ is noted $[a]_{\sim}$, and the quotient set of $\mathrm{A}$ with respect to $\sim$ is noted $\mathrm{A} / \sim$.

\section{B. Rigid transformations}

1) Continuous case: In $\mathbb{R}^{2}$, a rigid transformation is a function

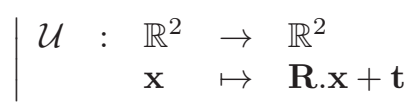

where $\mathbf{R}$ is a rotation matrix, and $\mathbf{t} \in \mathbb{R}^{2}$. The function $\mathcal{U}$ is a bijection, and we note $\mathcal{T}=\mathcal{U}^{-1}$ its inverse function, which is also a rigid transformation. We note $\mathfrak{R i g}_{\mathbb{R}^{2}}$ the set of the rigid transformations. 
2) Discrete case: These definitions cannot be directly applied in the discrete case, i.e., when considering $\mathbb{Z}^{2}$ instead of $\mathbb{R}^{2}$. Indeed, there is no guarantee that $\mathcal{U}\left(\mathbb{Z}^{2}\right) \subseteq \mathbb{Z}^{2}$. The handling of discrete rigid transformations then requires to consider a discretisation operator $\mathcal{D}: \mathbb{R}^{2} \rightarrow \mathbb{Z}^{2}$. In the most common cases -and in the present one- $\mathcal{D}$ is the standard rounding function. We can then define the discrete analogues $U: \mathbb{Z}^{2} \rightarrow \mathbb{Z}^{2}$ and $T: \mathbb{Z}^{2} \rightarrow \mathbb{Z}^{2}$, of $\mathcal{U}$ and $\mathcal{T}$, as

$$
\begin{aligned}
& U=\mathcal{D} \circ \mathcal{U}_{\mid \mathbb{Z}^{2}} \\
& T=\mathcal{D} \circ \mathcal{T}_{\mid \mathbb{Z}^{2}}=D \circ\left(\mathcal{U}^{-1}\right)_{\mid \mathbb{Z}^{2}}
\end{aligned}
$$

We note $\mathfrak{R i g}_{\mathbb{Z}^{2}}$ the set of the discrete rigid transformations.

3) Transformation models: Two transformation models can be considered for discrete (rigid) transformations: the Eulerian (or backwards) model, and the Lagragian (or forwards) one.

The Lagrangian model consists of computing $U\left(\mathbb{Z}^{2}\right)$, i.e., it determines the image of the "initial" space $\mathbb{Z}^{2}$ associated to the rigid transformation. From an imaging viewpoint, this model is not satisfactory, since $U$ is, in most cases, neither injective nor surjective. In other words, if $U$ is applied on a digital image (see Sec. II-C), it may lead to a transformed image that will present both undefined and conflicted values.

By opposition, the Eulerian model consists of computing $T\left(\mathbb{Z}^{2}\right)$, i.e., it determines the preimage of the "transformed" space $\mathbb{Z}^{2}$ associated to the rigid transformation. From an imaging viewpoint, this model is more satisfactory, since $T$ is defined on the whole transformed space $\mathbb{Z}^{2}$, thus guaranteeing that any point of a transformed digital image will be unambiguously defined. Nevertheless, since $T$ presents the same properties as $U$ in terms of non-injectivity and non-surjectivity, this model is not exempt from (topological) drawbacks.

\section{Digital images}

In this article, we consider finite digital images, that are defined as functions from $\mathbb{Z}^{2}$ to a value set $\mathbb{V}$. A digital image $I: \mathbb{Z}^{2} \rightarrow \mathbb{V}$ is considered as finite if there exists a value $\perp \in \mathbb{V}$ such that $I^{-1}(\mathbb{V} \backslash\{\perp\})$ is finite. The infinite part $I^{-1}(\{\perp\})$ is then considered as the "background" of the image. This assumption is motivated by practical considerations related to the digital definition of images in computer-based applications.

Still motivated by practical considerations, we consider three kinds of frequently used value sets for $\mathbb{V}$ :

- $\mathbb{B}=\{0,1\}$;

- $\mathbb{G} \subseteq \mathbb{Z}$ or $\mathbb{R}$ (equipped with the canonical order $\leqslant$ );

- $\mathbb{L}$, being any arbitrary set (non-equipped with an order).

The first case $(\mathbb{V}=\mathbb{B})$ deals with binary images. The set of finite binary images is noted $\mathfrak{I m}_{\mathbb{B}}$. The second case $(\mathbb{V}=\mathbb{G})$ deals with grey-level images. Without loss of generality, we can assume that $\perp=\bigwedge^{\leqslant} \mathbb{G}$. The set of finite grey-level images is noted $\mathfrak{I m}_{\mathbb{G}}$. The third case $(\mathbb{V}=\mathbb{L})$ deals with label images. The set of finite label images is noted $\mathfrak{I m}_{\mathbb{L}}$.

Remark 1: For the sake of readability, a point $\mathbf{p}=(x, y) \in$ $\mathbb{Z}^{2}$ will be associated to the pixel $\left[x-\frac{1}{2}, x+\frac{1}{2}\right] \times\left[y-\frac{1}{2}, y+\right.$ $\left.\frac{1}{2}\right] \subset \mathbb{R}^{2}$. In particular, the figures that illustrate the following sections rely on this digital interpretation.

\section{Digital topology}

1) Basic notions: Digital topology [16] provides a simple framework for handling the topology of binary images in $\mathbb{Z}^{n}$. Beyond its simplicity, it is also a robust framework that has been proved to be compliant [17] with other discrete models (e.g., Khalimsky grids [18] and cubical complexes [19]) but also with continuous notions of topology [20].

Practically, digital topology on $\mathbb{Z}^{n}$ mainly relies on two adjacency relations, noted $\frown_{2 n}$ and $\frown_{3^{n}-1}$, defined, for any $\mathbf{p}, \mathbf{q} \in \mathbb{Z}^{n}$, by

$$
\begin{aligned}
& \left(\mathbf{p} \frown_{2 n} \mathbf{q}\right) \Longleftrightarrow\left(\|\mathbf{p}-\mathbf{q}\|_{1}=1\right) \\
& \left(\mathbf{p} \frown 3^{n}-1 \mathbf{q}\right) \Longleftrightarrow\left(\|\mathbf{p}-\mathbf{q}\|_{\infty}=1\right)
\end{aligned}
$$

In the case of $\mathbb{Z}^{2}$, we retrieve in particular the well-known 4and 8 -adjacency relations, namely $\frown_{4}$ and $\frown_{8}$.

Let $\Omega \subseteq \mathbb{Z}^{2}$, and $\mathbf{p}, \mathbf{q} \in \Omega$. We say that $\mathbf{p}, \mathbf{q}$ are 4 - (resp. 8-) adjacent (in $\Omega$ ) if $\mathbf{p} \frown_{4} \mathbf{q}$ (resp. $\mathbf{p} \frown_{8} \mathbf{q}$ ). From the reflexivetransitive closure of $\frown_{4}$ (resp. $\left.\frown_{8}\right)$ on $\Omega$, we derive the 4 (resp. 8-) connectedness relation $\sim_{4}\left(\right.$ resp. $\left.\sim_{8}\right)$ (on $\Omega$ ); we say that $\mathbf{p}, \mathbf{q}$ are 4 - (resp. 8-) connected (in $\Omega$ ) if $\mathbf{p} \sim_{4} \mathbf{q}$ (resp. $\mathbf{p} \sim_{8} \mathbf{q}$ ). It is plain that $\sim_{4}$ (resp. $\sim_{8}$ ) is an equivalence relation on $\Omega$; the equivalence classes of $\Omega$ with respect to $\sim_{4}$ (resp. $\sim_{8}$ ), namely the elements of $\Omega / \sim_{4}$ (resp. $\Omega / \sim_{8}$ ) are called the 4- (resp. 8-) connected components of $\Omega$.

2) Dual adjacency and well-composedness models: A finite set $\Omega \subset \mathbb{Z}^{2}$ can be modeled as a binary image $I \in \mathfrak{I m}_{\mathbb{B}}$, defined by $I^{-1}(\{1\})=\Omega$ and $I^{-1}(\{0\})=\bar{\Omega}=\mathbb{Z}^{2} \backslash \Omega$, or vice versa. The topological handling of such a binary image cannot relevantly rely on $\frown_{8}$ for both $\Omega$ and $\bar{\Omega}$, due to paradoxes related to the discrete version of the Jordan theorem [21]. In this context, it has been proved [22] that such paradoxes could be avoided by considering distinct adjacencies for $\Omega$ and $\bar{\Omega}$, leading to the dual adjacency model (see Fig. 1(a-d)).

Definition 2 (Dual adjacency [22]): Let $I \in \mathfrak{I m}_{\mathbb{B}}$. Let $\Omega=$ $I^{-1}(\{1\})$ and $\bar{\Omega}=I^{-1}(\{0\})$. We say that $I$ is a $(8,4)$ - (resp. a $(4,8)$-) image if $\Omega$ is equipped with $\frown_{4}$ (resp. $\frown_{8}$ ), while $\bar{\Omega}$ is equipped with $\frown_{8}$ (resp. $\left.\frown_{4}\right)$. We define the set of the connected components of the $(8,4)$ - (resp. $(4,8)$-) image $I$ as

$$
\begin{aligned}
\mathcal{C}^{(8,4)}[I] & =I^{-1}(\{1\}) / \sim_{8} \cup I^{-1}(\{0\}) / \sim_{4} \\
\text { (resp. } \mathcal{C}^{(4,8)}[I] & \left.=I^{-1}(\{1\}) / \sim_{4} \cup I^{-1}(\{0\}) / \sim_{8}\right)
\end{aligned}
$$

(For the sake of concision, we will often write $(k, \bar{k})$ as a unified notation for $(8,4)$ and $(4,8)$.)

Alternatively, both $\Omega$ and $\bar{\Omega}$ may be equipped with $\frown_{4}$. In this context, it has been proposed [23] to only focus on images that avoid the issues related to the Jordan theorem, i.e., those for which $\sim_{4}$ and $\sim_{8}$ are equivalent for both $\Omega$ and $\bar{\Omega}$, thus leading to the well-composedness model (see Fig. 1(e-h)).

Definition 3 (Well-composedness [23]): Let $I \in \mathfrak{I m}_{\mathbb{B}}$. We say that $I$ is a well-composed (or a wc-) image if

$$
\forall v \in \mathbb{B}, I^{-1}(\{v\}) / \sim_{8}=I^{-1}(\{v\}) / \sim_{4}
$$

We define the set of the connected components of the $w c$ image $I$ as

$$
\mathcal{C}^{w c}[I]=I^{-1}(\{1\}) / \sim_{4} \cup I^{-1}(\{0\}) / \sim_{4}
$$




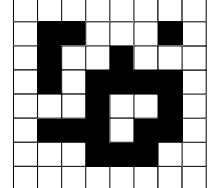

(a) $I_{1} \in \mathfrak{I m}_{\mathbb{B}}$

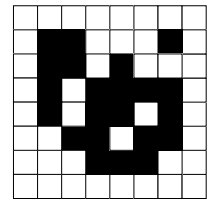

(e) $I_{2} \in \mathfrak{I m}_{\mathbb{B}}$

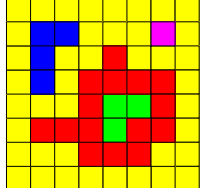

(b) $\mathcal{C}^{(8,4)}\left[I_{1}\right]$

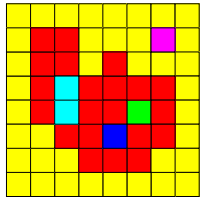

(f) $\mathcal{C}^{(8,4)}\left[I_{2}\right]$

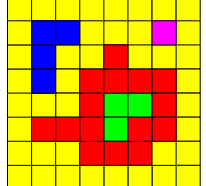

(c) $\mathcal{C}^{(4,8)}\left[I_{1}\right]$

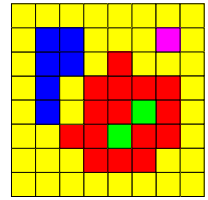

(g) $\mathcal{C}^{(4,8)}\left[I_{2}\right]$

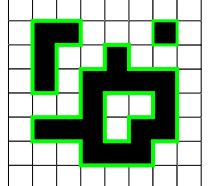

(d) $I_{1} \in \mathfrak{W C C}_{\mathbb{B}}$

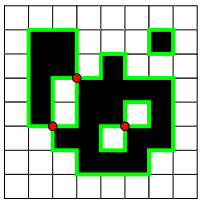

(h) $I_{2} \notin \mathfrak{W} \mathfrak{C C}_{\mathbb{B}}$
Fig. 1. (a) An image $I_{1} \in \mathfrak{I m}_{\mathbb{B}}$. (b) The 8-connected components of $\Omega=I_{1}^{-1}(\{1\})$ and the 4-connected components of $\bar{\Omega}=I_{1}^{-1}(\{0\})$. (c) The 4-connected components of $\Omega$ and the 8-connected components of $\bar{\Omega}$. Note that $I_{1}$ has the same topological structure as a $(8,4)$ - and as a $(4,8)$-image. (d) Then, $I_{1}$ can also be considered in the well-composedness model: the boundaries shared by its foreground and background regions, depicted in green, are 1-manifolds. (e) An image $I_{2} \in \mathfrak{I m}_{\mathbb{B}}$. (f) The 8connected components of $\Omega=I_{2}^{-1}(\{1\})$ and the 4-connected components of $\bar{\Omega}=I_{2}^{-1}(\{0\})$. (g) The 4-connected components of $\Omega$ and the 8-connected components of $\bar{\Omega}$. Note that $I_{2}$ does not have the same topological structure as a $(8,4)$ - and as a $(4,8)$-image. (h) Then, $I_{2}$ cannot be considered in the well-composedness model: the boundaries shared by its foreground and background regions, depicted in green, are not 1-manifolds (see the red dots). (a,d,e,h) $\Omega$ is depicted in black, and $\bar{\Omega}$ in white. (b,c,f,g) For the sake of readability, each connected component is represented by a different colour.

The set of the finite well-composed binary images is noted $\mathfrak{W J C}_{\mathbb{B}}$.

Remark 4: When "interpreting" digital topology in a continuous framework [17], an image is well-composed iff the boundaries shared by the foreground and background regions are manifolds [23] (see Fig. 1(d,h)).

Remark 5: The well-composedness model is more restrictive than the dual adjacency one. Indeed, any $I \in \mathfrak{I m}_{\mathbb{B}}$ can be considered in the dual adjacency model, but not necessarily in the well-composedness one, i.e.

$$
\mathfrak{W} \mathfrak{C}_{\mathbb{B}} \subset \mathfrak{I m}_{\mathbb{B}}
$$

\section{PuRPOSE AND CHOICES}

Let us consider an image $I \in \mathfrak{I m}_{\mathbb{V}}$, a transformation $T$ : $\mathbb{Z}^{2} \rightarrow \mathbb{Z}^{2}$, and the transformed image $I_{T} \in \mathfrak{I m}_{\mathbb{V}}$ obtained from $I$ and $T$. A frequent question in image analysis is: "Does $T$ preserve the topology between $I$ and $I_{T}$ ?" It is generally answered by considering topological invariants of the images.

The most simple ones are, e.g., the Euler-Poincaré characteristic, or the Betti Numbers. However, they are too weak to correctly model the "topology preservation" between images. It is then mandatory to consider stronger topological invariants, e.g., the (digital) fundamental group [24], the homotopy-type (considered via notions of simple points/sets [25], [26], [27], [28]), or the adjacency-tree [29].

Our first goal is to provide conditions under which 2D digital images preserve their topological properties under any rigid transformation. In this context, a crucial issue is the choice of the topological invariant used to formalise this problem. Any of those presented above describe topology preservation in a global fashion, and do not model accurately the possible local modifications of the image topological structure. Broadly speaking, $I$ and $I_{T}$ may have the same fundamental group, homotopy-type and/or adjacency-tree while there exist some topological differences between some regions of $I$ and $I_{T}$ that are in correspondence with respect to $T$ (see, e.g., [30]).

In the sequel, we propose some conditions that reach that first goal. Our conjecture is that these conditions are necessary and sufficient to locally preserve image topological properties under any rigid transformation. However, in this article, we only establish that they are sufficient to globally preserve image topological properties under any rigid transformation.

Indeed, on the one hand, the proof of the whole conjecture would require to develop a heavy theoretical framework, that falls out of the scope of this journal (see Sec.VI). On the other hand, the sufficiency of these conditions is the part of the result that is actually useful to justify and develop methodological tools for image (pre)processing, that is the second purpose of this article, and probably the most interesting for the reader.

We will consider, as (global) topological invariant, the adjacency-tree [29]. The motivation of this choice is twofold: ( $i$ ) the understanding of this topological invariant is probably easier for most readers; and $(i i)$ in the $2 \mathrm{D}$ case, its preservation is equivalent [31] to the preservation of the homotopy-type, that is the most commonly used topological invariant in image processing. We now recall the definition of the adjacency-tree.

Let $I \in \mathfrak{I m}_{\mathbb{B}}\left(\right.$ resp. $\mathfrak{W C}_{\mathbb{B}}$ ). Let $\Omega_{1}, \Omega_{2} \in \mathcal{C}^{(k, \bar{k})}[I]$ (resp. $\mathcal{C}^{w c}[I]$ ), with $\Omega_{1} \neq \Omega_{2}$. We note $\Omega_{1} \frown_{I}^{(k, \bar{k})} \Omega_{2}$ (resp. $\Omega_{1} \frown_{I}^{w c}$ $\Omega_{2}$ ) if there exist $\mathbf{p}_{\in} \in \Omega_{1}$ and $\mathbf{q} \in \Omega_{2}$ such that $\mathbf{p} \frown_{4} \mathbf{q}$. It is plain that $\frown_{I}^{(k, \bar{k})}$ (resp. $\frown_{I}^{w c}$ ) is an adjacency relation, and that $\Omega_{1} \frown_{I}^{(k, \bar{k})} \Omega_{2}$ implies that $\Omega_{1} \in I^{-1}(\{1\}) / \sim_{k}$ and $\Omega_{2} \in I^{-1}(\{0\}) / \sim_{\bar{k}}$ or vice versa. We define the $(k, \bar{k})$ - (resp. wc-) adjacency graph of $I$ as $\mathfrak{G}^{(k, \bar{k})}(I)=\left(\mathcal{C}^{(k, \bar{k})}[I], \frown_{I}^{(k, \bar{k})}\right)$ (resp. $\left.\mathfrak{G}^{w c}(I)=\left(\mathcal{C}^{w c}[I], \frown_{I}^{w c}\right)\right)$. This graph is connected and acyclic, and is indeed a tree. It can be equipped with a root that is the (only) infinite connected component of $\mathcal{C}^{(k, \bar{k})}[I]$ (resp. $\mathcal{C}^{w c}[I]$ ), thus leading to the following definition.

Definition 6 (Adjacency tree [29]): Let $I \in \mathfrak{I m}_{\mathbb{B}}$ (resp. $\left.\mathfrak{W C}_{\mathbb{B}}\right)$. The $(k, \bar{k})$ - (resp. wc-) adjacency tree of $I$ is the triple

$$
\begin{aligned}
\mathfrak{T}^{(k, \bar{k})}(I) & =\left(\mathcal{C}^{(k, \bar{k})}[I], \frown_{I}^{(k, \bar{k})}, B_{I}^{(k, \bar{k})}\right) \\
\text { (resp. } \mathfrak{T}^{w c}(I) & \left.=\left(\mathcal{C}^{w c}[I], \frown_{I}^{w c}, B_{I}^{w c}\right)\right)
\end{aligned}
$$

where $B_{I}^{(k, \bar{k})} \in \mathcal{C}^{(k, \bar{k})}[I]$ (resp. $B_{I}^{w c} \in \mathcal{C}^{w c}[I]$ ) is the unique infinite connected component of $I$.

We are now ready to present our definition of topologypreservation under rigid transformation.

Definition 7 (Topological invariance): Let $I \in \mathfrak{I m}_{\mathbb{B}}$ (resp. $\mathfrak{W C}_{\mathbb{B}}$ ). We say that $I$ is $(k, \bar{k})$ - (resp. wc-) topologically invariant if any $T \in \mathfrak{R i g}_{\mathbb{Z}^{2}}$ induces an isomorphism between $\mathfrak{T}^{(k, \bar{k})}(I)\left(\operatorname{resp} . \mathfrak{T}^{w c}(I)\right)$ and $\mathfrak{T}^{(k, \bar{k})}(I \circ T)\left(\operatorname{resp} . \mathfrak{T}^{w c}(I \circ T)\right)$, and if $I \circ T \in \mathfrak{I m}_{\mathbb{B}}$ (resp. $\mathfrak{W J C}_{\mathbb{B}}$ ). We note $\mathfrak{I n v}_{\mathbb{B}}^{(k, \bar{k})}$ (resp. $\left.\mathfrak{I n v}_{\mathbb{B}}^{w c}\right)$ the set of the $(k, \bar{k})$ - (resp. $\left.w c-\right)$ topologically invariant binary images. 


\section{TheoreticAl Results}

In this section, we define a notion of regularity (Sec. IV-C) that provides conditions under which binary images are topologically invariant (Sec. IV-D). We then derive analogue conditions for grey-level (Sec. IV-E) and label images (Sec. IV-F).

\section{A. Preliminary remarks}

As stated above, we consider the Eulerian transformation model (Sec. II-B), and we first focus on binary images. In other words, given an image $I \in \mathfrak{I m}_{\mathbb{B}}$ and a discrete rigid transfomation $T \in \mathfrak{R i g}_{\mathbb{Z}^{2}}$ (intrinsically associated to a rigid transformation $\mathcal{T} \in \mathfrak{R i g}_{\mathbb{R}^{2}}$ ), we consider the transformed image $I_{T} \in \mathfrak{I m}_{\mathbb{B}}$ defined as

$$
I_{T}=I \circ T=I \circ \mathcal{D} \circ \mathcal{T}_{\mid \mathbb{Z}^{2}}
$$

By setting $\Omega=I^{-1}(\{1\}), \bar{\Omega}=I^{-1}(\{0\})$ and $\Omega_{T}=$ $I_{T}^{-1}(\{1\}), \overline{\Omega_{T}}=I_{T}^{-1}(\{0\})$, Eq. (13) rewrites as

$$
\begin{aligned}
& \Omega_{T}=\mathbb{Z}^{2} \cap \mathcal{T}^{-1}(\Omega \oplus \square) \\
& \overline{\Omega_{T}}=\mathbb{Z}^{2} \cap \mathcal{T}^{-1}(\bar{\Omega} \oplus \square)
\end{aligned}
$$

where $\oplus$ is the dilation operator defined in mathematical morphology (see, e.g., [32]-Ch. 1), and $\square \subset \mathbb{R}^{2}$ is the unit square, namely a pixel. These equations can lead to different results depending on the definition of this pixel, that may be $\square=\left[-\frac{1}{2}, \frac{1}{2}\right]^{2}$ or $]-\frac{1}{2}, \frac{1}{2}[2$. This motivates the next remark.

Remark 8: In this work, we assume that $\mathcal{T}$ and $T$ are such that $\mathbb{Z}^{2}$ does not intersect any transformed pixel border. In other words, we consider that Eqs. (13)-(15) lead to equal results for both definitions of $\square$. From a theoretical viewpoint, this allows us to develop a general discussion without confusing variants related to $\mathcal{D}$. From a pratical viewpoint, this assumption is compliant with computer-based applications, that generally rely on floating point arithmetic.

\section{B. Image space restrictions}

We first state that the binary images considered for the study of topological invariance can be chosen in a subspace of $\mathfrak{I m}_{\mathbb{B}}$.

Remark 9: We restrict our study of $(k, \bar{k})$-topological invariance within the binary images to the subspace $\mathfrak{W} \mathfrak{C}_{\mathbb{B}} \subset$ $\mathfrak{I m}_{\mathbb{B}}$. This restriction is motivated ${ }^{1}$ by the fact that any $I \in \mathfrak{I m}_{\mathbb{B}} \backslash \mathfrak{W J C}_{\mathbb{B}}$ presents configurations (see Th. 23) that may be non-compliant with the definition of $(k, \bar{k})$-topological invariance.

We now introduce a notion of singularity, and we establish that singular images cannot be topologically invariant, thus reducing the image subspace to consider.

Definition 10 ((Non-)singular image): Let $I \in \mathfrak{I m}_{\mathbb{B}}$. We say that $I$ is a singular image if

$$
\exists \mathbf{p} \in \mathbb{Z}^{2}, \forall \mathbf{q} \in \mathbb{Z}^{2},\left(\mathbf{q} \frown_{4} \mathbf{p}\right) \Longrightarrow(I(\mathbf{p}) \neq I(\mathbf{q}))
$$

We note $\mathfrak{N S}_{\mathbb{B}}$ the set of the well-composed images that are not singular.

\footnotetext{
${ }^{1}$ This restriction, presented as a motivated -but arbitrary- choice when considering a global topological invariant, may however be proved when considering a local one. As discussed in Sec. III, such a proof is beyond the scope of this article.
}

Proposition 11: We have

$$
\mathfrak{I n} \mathfrak{v}_{\mathbb{B}}^{w c} \subseteq\left(\mathfrak{I n} \mathfrak{v}_{\mathbb{B}}^{(k, \bar{k})} \cap \mathfrak{W} \mathfrak{C}_{\mathbb{B}}\right) \subseteq \mathfrak{N} \mathfrak{S}_{\mathbb{B}}
$$

In the sequel, we then carry out our study of topological invariance within the set of well-composed non-singular images, independently from the considered (dual adjacency, or wellcomposedness) model.

\section{Regularity}

Let us now introduce a new notion that strenghtens the notion of well-composedness.

Definition 12 (Regularity): Let $I \in \mathfrak{N S}_{\mathbb{B}}$. We say that $I$ is $k$-regular (resp. $\bar{k}$-regular) if for any $\mathbf{p}, \mathbf{q} \in I^{-1}(\{1\})$ (resp. $\left.I^{-1}(\{0\})\right)$, we have

$$
\begin{aligned}
\left(\mathbf{p} \frown_{4} \mathbf{q}\right) & \Longrightarrow \exists \boxplus \subseteq I^{-1}(\{1\}), \mathbf{p}, \mathbf{q} \in \boxplus \\
\left(\text { resp. }\left(\mathbf{p} \frown_{4} \mathbf{q}\right)\right. & \left.\Longrightarrow \exists \boxplus \subseteq I^{-1}(\{0\}), \mathbf{p}, \mathbf{q} \in \boxplus\right)
\end{aligned}
$$

where $\boxplus$ is a "square" element, i.e., $\boxplus=\{x, x+1\} \times\{y, y+1\}$, for $(x, y) \in \mathbb{Z}^{2}$. We say that $I$ is regular if it is both $k$ - and $\bar{k}$-regular. We note $\mathfrak{R e} \mathfrak{g}_{\mathbb{B}}^{k}$ (resp. $\mathfrak{R e} \mathfrak{g}_{\mathbb{B}}^{\bar{k}}$, resp. $\mathfrak{R e} \mathfrak{g}_{\mathbb{B}}$ ) the set of the $k$-regular (resp. $\bar{k}$-regular, resp. regular) binary images.

Remark 13: Following mathematical morphology terminology and notations (see, e.g., [32]-Ch. 1), if $I$ is $k$ - (resp. $\bar{k}$-) regular, then $\Omega=I^{-1}(\{1\})$ (resp. $\Omega=I^{-1}(\{0\})$ ) is open by any structuring element $\boxplus$, i.e.

$$
\gamma_{\boxplus}(\Omega)=\Omega \ominus \boxplus \oplus \boxplus=\Omega
$$

\section{Topological invariance: the binary case}

We now establish our main result for binary images, that states that regularity implies topological invariance.

Theorem 14:

$$
\begin{aligned}
& \mathfrak{R} \mathfrak{e} \mathfrak{g}_{\mathbb{B}}^{\bar{k}} \subseteq \mathfrak{I} \mathfrak{n} \mathfrak{v}_{\mathbb{B}}^{(8,4)} \\
& \mathfrak{R} \mathfrak{e} \mathfrak{g}_{\mathbb{B}}^{k} \subseteq \mathfrak{I} \mathfrak{n} \mathfrak{v}_{\mathbb{B}}^{(4,8)} \\
& \mathfrak{R} \mathfrak{e} \mathfrak{g}_{\mathbb{B}} \subseteq \mathfrak{I n} \mathfrak{v}_{\mathbb{B}}^{w c}
\end{aligned}
$$

\section{E. Topological invariance: the grey-level case}

In Sec. II-C, it has been observed that a grey-level image takes its values in a finite (and totally ordered) subset of $\mathbb{Z}$ or $\mathbb{R}$. Any such image is then equivalent to an image $I: \mathbb{Z}^{2} \rightarrow \mathbb{G}$, where $\mathbb{G}=\llbracket 0, m \rrbracket \subset \mathbb{Z}$ is a finite interval, and $\perp=0$. Without loss of generality, we then focus on such images.

A grey-level image $I \in \mathfrak{I m}_{\mathbb{G}}$ can unambiguously be modeled by the finite set of its binary level set images $\lambda_{v}(I) \in \mathfrak{I m}_{\mathbb{B}}$ defined, for any $v \in \mathbb{G}$ as

$$
\begin{aligned}
\lambda_{v}(I): \mathbb{Z} & \rightarrow \mathbb{B} \\
\mathbf{p} & \mapsto \begin{cases}1 & \text { if } v \leqslant I(\mathbf{p}) \\
0 & \text { otherwise }\end{cases}
\end{aligned}
$$

The image $I$ can then be reconstructed as the supremum of these $|\mathbb{G}|$ level set images, with respect to the pointwise order $\leq$ on functions induced by the order $\leqslant$ on $\mathbb{G}$

$$
I=\bigvee_{v \in \mathbb{V}}^{\leq} v \cdot \lambda_{v}(I)
$$


Based on this modelling of $I \in \mathfrak{I m}_{\mathbb{G}}$ by the set $\left\{\lambda_{v}(I)\right\}_{v \in \mathbb{G}}$, the notions previously introduced for binary images can be extended to grey-level ones ${ }^{2}$. In particular, we have

$$
\begin{aligned}
& \mathfrak{W C}_{\mathbb{G}}=\left\{I \in \mathfrak{I m}_{\mathbb{G}} \mid \forall v \in \mathbb{G}, \lambda_{v}(I) \in \mathfrak{W C}_{\mathbb{B}}\right\} \\
& \mathfrak{N S}_{\mathbb{G}}=\left\{I \in \mathfrak{W C}_{\mathbb{G}} \mid \forall v \in \mathbb{G}, \lambda_{v}(I) \in \mathfrak{N S _ { \mathbb { B } }}\right\}
\end{aligned}
$$

Moreover, we define the analogues of the binary notions of topological invariance (Def. 7) and regularity (Def. 12).

Definition 15 (Grey-level topological invariance): Let $I \in$ $\mathfrak{N S}_{\mathbb{G}}$. We say that $I$ is $(k, \bar{k})$ - (resp. wc-) topologically invariant if for any $v \in \mathbb{G}, \lambda_{v}(I) \in \mathfrak{I n v}_{\mathbb{B}}^{(k, \bar{k})}$ (resp. $\mathfrak{I n v}_{\mathbb{B}}^{w c}$ ). We note $\mathfrak{I n v}_{\mathbb{G}}^{(k, \bar{k})}\left(\right.$ resp. $\left.\mathfrak{I n v}_{\mathbb{G}}^{w c}\right)$ the set of the $(k, \bar{k})$ - (resp. $w c-)$ topologically invariant grey-level images.

Definition 16 (Grey-level regularity): Let $I \in \mathfrak{N S}_{\mathbb{G}}$. We say that $I$ is $k$-regular (resp. $\bar{k}$-regular, resp. regular) if for any $v \in \mathbb{G}, \lambda_{v}(I) \in \mathfrak{R e} \mathfrak{g}_{\mathbb{B}}^{k}$ (resp. $\mathfrak{R} \mathfrak{e} \mathfrak{g}_{\mathbb{B}}^{\bar{k}}$, resp. $\mathfrak{R e} \mathfrak{g}_{\mathbb{B}}$ ). We note $\mathfrak{R e} \mathfrak{g}_{\mathbb{G}}^{k}$ (resp. $\mathfrak{R} \mathfrak{e} \mathfrak{g}_{\mathbb{G}}^{k}$, resp. $\mathfrak{R} \mathfrak{e} \mathfrak{g}_{\mathbb{G}}$ ) the set of the $k$-regular (resp. $\bar{k}$-regular, resp. regular) grey-level images.

The following theorem, that is the grey-level analogue of Th. 14, straightforwardly derives from this last theorem, and Defs. $15,16$.

Theorem 17:

$$
\begin{aligned}
& \mathfrak{R e} \mathfrak{g}_{\mathbb{G}}^{\bar{k}} \subseteq \mathfrak{I} \mathfrak{n} \mathfrak{v}_{\mathbb{G}}^{(8,4)} \\
& \mathfrak{R e} \mathfrak{g}_{\mathbb{G}}^{k} \subseteq \mathfrak{I} \mathfrak{n} \mathfrak{v}_{\mathbb{G}}^{(4,8)} \\
& \mathfrak{R e} \mathfrak{g}_{\mathbb{G}} \subseteq \mathfrak{I n} \mathfrak{v}_{\mathbb{G}}^{w c}
\end{aligned}
$$

Remark 18: The topological invariance (and thus, the regularity) of $I \in \mathfrak{I m}_{\mathbb{G}}$ also leads to the preservation of the hierarchy of its connected components between successive levels. More precisely, the $(k, \bar{k})$ - (resp. wc-) topological invariance implies that for any $T \in \mathfrak{R i g}_{\mathbb{Z}^{2}}$, the images $I$ and $I \circ T$ have isomorphic component-trees [34]. This assertion is easy to prove, based on the fact that $(i) T$ establishes a bijection between the connected components of the initial and transformed level set images (Prop. 35), and (ii) $T$ preserves, by construction (see Eqs. (3), (24)-(25)), the inclusion relation between these components at successive levels. A more complete discussion on this topic is beyond the scope of this article; the reader is referred, e.g., to [34], [35] or [32]-Ch. 7 (and the references therein) for complementary information.

\section{F. Topological invariance: the label case}

Similarly to the case of grey-level images, it has been observed in Sec. II-C that a label image takes its values in a finite set. Any such image is then equivalent to an image $I: \mathbb{Z}^{2} \rightarrow \mathbb{L}$, where $\mathbb{L}$ is finite and $\perp \in \mathbb{L}$. Without loss of generality, we now focus on such images.

Several attempts have been made to propose topological frameworks for label images, and more precisely to define what is the exact meaning of the "topology preservation" in such images [36], [37], [5], [38]. In this work, we follow a recent and general proposal [39], [40], that consists of considering the values of $\mathbb{L}$ as "proto-labels", and any subsets of such values as the actual labels of the image. In other words,

${ }^{2}$ A notion of grey-level well-composedness has also been proposed in [33]. the topology of a label image $I \in \mathfrak{I m}_{\mathbb{L}}$, and its potential preservation, are considered by observing all the binary images exhaustively induced by labels $\Lambda$ that are the elements of the power set $2^{\mathbb{L}}$. This leads to the following notions.

A label image $I \in \mathfrak{I m}_{\mathbb{L}}$ can unambiguously be modeled by the finite set of its binary characteristic images $\chi_{\Lambda}(I) \in \mathfrak{I m}_{\mathbb{B}}$ defined, for any $\Lambda \in 2^{\mathbb{L}}$ as

$$
\begin{aligned}
\chi_{\Lambda}(I): \mathbb{Z} & \rightarrow \mathbb{B} \\
\mathbf{p} & \mapsto \begin{cases}1 & \text { if } I(\mathbf{p}) \in \Lambda \\
0 & \text { otherwise }\end{cases}
\end{aligned}
$$

In particular, by identifying $(i)$ the sets $\{l\}_{l \in \mathbb{L}}$ and $\{\{l\}\}_{l \in \mathbb{L} \text {, }}$ and $(i i)$ the monoids $(\mathbb{B},$.$) and (\{\mathbb{L}, \emptyset\}, \cup)$, the image $I$ can be reconstructed -similarly to the case of grey-level images (see Eq. (25))- as the infimum of these $2^{|\mathbb{L}|}$ characteristic images, with respect to the pointwise order $\sqsubseteq$ on functions induced by the inclusion order $\subseteq$ on $2^{\mathbb{L}}$

$$
I=\bigwedge_{\Lambda \in 2^{\mathbb{L}}}^{\check{c}} \Lambda \cdot \chi_{\Lambda}(I)
$$

Based on this modelling of $I \in \mathfrak{I m}_{\mathbb{L}}$ by the set $\left\{\chi_{\Lambda}(I)\right\}_{\Lambda \in 2^{\amalg}}$, the notions previously introduced for binary images can be extended ${ }^{3}$ to label ones. In particular, we have

$$
\begin{aligned}
& \mathfrak{W C}_{\mathbb{L}}=\left\{I \in \mathfrak{I m}_{\mathbb{L}} \mid \forall \Lambda \in 2^{\mathbb{L}}, \chi_{\Lambda}(I) \in \mathfrak{W C}_{\mathbb{B}}\right\} \\
& \mathfrak{N S}_{\mathbb{L}}=\left\{I \in \mathfrak{W C}_{\mathbb{L}} \mid \forall \Lambda \in 2^{\mathbb{L}}, \chi_{\Lambda}(I) \in \mathfrak{N S _ { \mathbb { B } }}\right\}
\end{aligned}
$$

Moreover, we define the analogues of the binary notions of topological invariance (Def. 7) and regularity (Def. 12).

Definition 19 (Label topological invariance): Let

$I \in \mathfrak{N S}_{\mathbb{L}}$. We say that $I$ is $(k, \bar{k})$ - (resp. wc-) topologically invariant if for any $\Lambda \in 2^{\mathbb{L}}, \chi_{\Lambda}(I) \in \mathfrak{I n v}_{\mathbb{B}}^{(k, \bar{k})}$ (resp. $\mathfrak{I n} \mathfrak{v}_{\mathbb{B}}^{w c}$ ). We note $\mathfrak{I n v}_{\mathbb{L}}^{(k, \bar{k})}\left(\right.$ resp. $\mathfrak{I n v}_{\mathbb{L}}^{w c}$ ) the set of the $(k, \bar{k})$ - (resp. $w c$-) topologically invariant label images.

Remark 20: In the sequel, we restrict ${ }^{4}$ our study to the case of $w c$-topological invariance for label images.

Definition 21 (Label regularity): Let $I \in \mathfrak{N S}_{\mathbb{L}}$. We say that $I$ is regular if for any $\Lambda \in 2^{\mathbb{L}}, \chi_{\Lambda}(I) \in \mathfrak{R e} \mathfrak{g}_{\mathbb{B}}$. We note $\mathfrak{R e} \mathfrak{g}_{\mathbb{L}}$ the set of regular label images.

The following theorem, that is the label analogue of Th. 14, straightforwardly derives from this last theorem, and Defs. 19, 21.

Theorem 22:

$$
\mathfrak{R e} \mathfrak{g}_{\mathbb{L}} \subseteq \mathfrak{I n} \mathfrak{v}_{\mathbb{L}}^{w c}
$$

\section{Methodogy}

In Sec. IV, we have established sufficient conditions for guaranteeing topological invariance, thanks to the notion of regularity (Ths. 14, 17, 22). From this theoretical study, we first propose simple algorithms to characterise the regularity of

\footnotetext{
${ }^{3}$ The definition of well-composedness for label images proposed here (see Eq. (33)) slightly differs from the one introduced in [36], that only requires that $\chi_{\{l\}}(I) \in \mathfrak{W C}_{\mathbb{B}}$ for any proto-label $l \in \mathbb{L}$.

${ }^{4} \mathrm{As}$ in Rem. 9 and the associated footnote, this restriction is motivated by the fact that the $(8,4)$ - and $(4,8)$-topological invariance (that are equal, from there very definitions) may be proved to be equal to the $w c$-topological invariance. Once again, the proof of this assertion is beyond the scope of this article.
} 


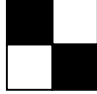

(a)

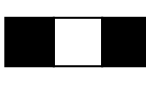

(b)

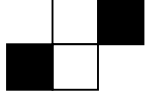

(c)
Fig. 2. Forbidden patterns in $\mathfrak{W J C}_{\mathbb{B}}$ (a) and in $\mathfrak{R e} \mathfrak{g}_{\mathbb{B}}^{\bar{k}}$ (a-c), up to $\pi / 2$ rotations and symmetries. The patterns forbidden in $\mathfrak{R e} \mathfrak{g}_{\mathbb{B}}^{k}$ are obtained from $(\mathrm{a}-\mathrm{c})$ by value inversion. Black (resp. white) points have value 1 (resp. 0).

an image (Sec. V-A). Then, we describe some preprocessing strategies that enable to turn a non-regular image into a regular (and then topologically invariant) one (Sec. V-B).

\section{A. Pattern-based characterisation of regular images}

In this section, we show that the regularity of a $2 \mathrm{D}$ digital image can be easily determined by considering a small set of specific patterns. This result straightforwardly leads to an algorithm of optimal time and space complexity.

1) Well-composedness characterisation: Regular images are defined within the set of well-composed ones. A prerequisite is then to characterise $\mathfrak{W J C}_{\mathbb{B}}$. This is tractable by considering a specific $2 \times 2$ pattern [23].

Theorem 23 ([23]): Let $I \in \mathfrak{I m}_{\mathbb{B}}$. We have $I \notin \mathfrak{W J C}_{\mathbb{B}}$ iff there exist distinct points $\mathbf{p}, \mathbf{q}, \mathbf{r}, \mathbf{s} \in \mathbb{Z}^{2}$, with $\mathbf{p} \frown_{4} \mathbf{q} \frown_{4}$ $\mathbf{r} \frown_{4} \mathbf{S} \frown_{4} \mathbf{p}$, that verify

$$
I(\mathbf{p}) \neq I(\mathbf{q}) \neq I(\mathbf{r}) \neq I(\mathbf{s})
$$

Based on Th. 23 and Defs. 15, 19, we straightforwardly derive characterisations of grey-level and label well-composedness.

Corollary 24: Let $I \in \mathfrak{I m}_{\mathbb{G}}$. We have $I \notin \mathfrak{W J C}_{\mathbb{G}}$ iff there exist distinct points $\mathbf{p}, \mathbf{q}, \mathbf{r}, \mathbf{s} \in \mathbb{Z}^{2}$, with $\mathbf{p} \frown_{4} \mathbf{q} \frown_{4} \mathbf{r} \frown_{4}$ $\mathbf{s} \frown_{4} \mathbf{p}$, that verify

$$
I(\mathbf{p})>I(\mathbf{q})<I(\mathbf{r})>I(\mathbf{s})<I(\mathbf{p})
$$

Corollary 25: Let $I \in \mathfrak{I m}_{\mathbb{L}}$. We have $I \notin \mathfrak{W J C}_{\mathbb{L}}$ iff there exist distinct points $\mathbf{p}, \mathbf{q}, \mathbf{r}, \mathbf{s} \in \mathbb{Z}^{2}$, with $\mathbf{p} \frown_{4} \mathbf{q} \frown_{4} \mathbf{r} \frown_{4}$ $\mathbf{s} \frown_{4} \mathbf{p}$, that verify

$$
I(\mathbf{p}) \neq I(\mathbf{q}) \neq I(\mathbf{r}) \neq I(\mathbf{s}) \neq I(\mathbf{p})
$$

The characterisation of binary, grey-level and label images as well-composed ones can then be carried out by simply checking that they do not contain the forbidden patterns induced by the binary pattern depicted in Fig. 2(a).

2) Regularity characterisation: We now propose a patternbased characterisation of regular binary images.

Proposition 26: Let $I \in \mathfrak{W C}_{\mathbb{B}}$. We have $I \notin \mathfrak{R e g} \mathfrak{g}_{\mathbb{B}}^{k}$ (resp. $\left.\mathfrak{R e} \mathfrak{g}_{\mathbb{B}}^{\bar{k}}\right)$, and a fortiori $\mathfrak{R e} \mathfrak{g}_{\mathbb{B}}$, iff there exists $\mathbf{p} \in I^{-1}(\{1\})$ (resp. $I^{-1}(\{0\})$ ) that satisfies at least one of the following two conditions (up to $\pi / 2$ rotations and symmetries)

$$
\begin{aligned}
& I(\mathbf{p}-(1,0)) \neq I(\mathbf{p}) \neq I(\mathbf{p}+(1,0)) \\
& I(\mathbf{p}+(0,1))=I(\mathbf{p}) \neq I(\mathbf{p}-(1,0))=I(\mathbf{p}+(1,1))
\end{aligned}
$$

Based on Prop. 26 and Defs. 15, 19, we straightforwardly derive characterisations of grey-level and label regularity.

Corollary 27: Let $I \in \mathfrak{W C}_{\mathbb{G}}$. We have $I \notin \mathfrak{R e g} \mathfrak{g}_{\mathbb{G}}^{k}$ (resp. $\left.\mathfrak{R e} \mathfrak{g}_{\mathbb{G}}^{\bar{k}}\right)$, and a fortiori $\mathfrak{R e} \mathfrak{g}_{\mathbb{G}}$, iff there exists $\mathbf{p} \in \mathbb{Z}^{2}$ that satisfies at least one of the following two conditions (up to $\pi / 2$ rotations and symmetries)

$$
\begin{aligned}
I(\mathbf{p}-(1,0))<I(\mathbf{p})>I(\mathbf{p}+(1,0)) \\
(\operatorname{resp.} I(\mathbf{p}-(1,0))>I(\mathbf{p})<I(\mathbf{p}+(1,0))) \\
I(\mathbf{p}+(0,1)) \geqslant I(\mathbf{p})>I(\mathbf{p}-(1,0)) \geqslant I(\mathbf{p}+(1,1)) \\
(\operatorname{resp.} I(\mathbf{p}+(0,1)) \leqslant I(\mathbf{p})<I(\mathbf{p}-(1,0)) \leqslant I(\mathbf{p}+(1,1)))
\end{aligned}
$$

Corollary 28: Let $I \in \mathfrak{W C}_{\mathbb{L}}$. We have $I \notin \mathfrak{R e} \mathfrak{g}_{\mathbb{L}}$ iff there exists $\mathbf{p} \in \mathbb{Z}$ that satisfies at least one of the following two conditions (up to $\pi / 2$ rotations and symmetries)

$$
\begin{aligned}
I(\mathbf{p}-(1,0)) & \neq I(\mathbf{p}) \neq I(\mathbf{p}+(1,0)) \\
I(\mathbf{p}) \neq I(\mathbf{p}-(1,0)) & \neq I(\mathbf{p}+(0,1)) \neq I(\mathbf{p}+(1,1)) \neq I(\mathbf{p})
\end{aligned}
$$

The characterisation of binary, grey-level and label images as regular ones can then be carried out by simply checking that they do not contain the forbidden patterns induced by the binary patterns depicted in Fig. 2(a-c).

3) Complexity: The following result straightforwardly derives from Th. 23, Prop. 26, and their respective corollaries.

Proposition 29: Let $I \in \mathfrak{W J C}_{\mathbb{V}}$ (with $\mathbb{V}=\mathbb{B}, \mathbb{G}$ or $\mathbb{L}$ ). Let $\mathbb{S} \subset \mathbb{Z}^{2}$ be such that $I^{-1}(\mathbb{V} \backslash\{\perp\}) \subseteq \mathbb{S}$ (practically, $\mathbb{S}$ is the finite set where $I$ is defined in a computer-based application). Then, the algorithm that determines the (non-)regularity of $I$, has a time complexity $\mathcal{O}(|\mathbb{S}|)$, and a space complexity $\mathcal{O}(1)$.

\section{B. Image regularisation}

We now propose two strategies for preprocessing images in order to obtain regular -and then topologically invariantones, before further rigid transformation. Such regularisation strategies $(i)$ must preserve the topological properties of the images, and (ii) should preserve as much as possible their geometric properties.

1) Iterative homotopic regularisation: A first strategy consists of locally modifying the image to eliminate the forbidden configurations defined in Eqs. (36)-(46) and Fig. 2.

Let $I \in \mathfrak{I m}_{\mathbb{V}}$ (or $\mathfrak{W C}_{\mathbb{V}}$, if we aim to obtain regularity, and not only $k$ or $\bar{k}$-one). The problem to tackle can be expressed as a constrained optimisation one, described by

$$
R(I)=\arg \min _{\mathfrak{R} \mathfrak{e} \mathfrak{g}_{\mathbb{V}}^{\star}(I)} D_{I}
$$

where $R(I)$ is the regularised version of $I ; \mathfrak{R e g}_{\mathbb{V}}^{\star}(I)$ is the subset of $\mathfrak{R e g} \mathfrak{g}_{\mathbb{V}}^{\star} \in\left\{\mathfrak{R e} \mathfrak{g}_{\mathbb{V}}^{k}, \mathfrak{R e} \mathfrak{g}_{\mathbb{V}}^{\bar{k}}, \mathfrak{R e} \mathfrak{g}_{\mathbb{V}}\right\}$ composed by the images that have the same topology as $I$; and $D_{I}: \mathfrak{I m}_{\mathbb{V}} \rightarrow \mathbb{R}_{+}$ is a cost function that describes the "distance" from $I$, from a geometric viewpoint. (The definition of $D_{I}$ actually depends on the targeted application, and can rely, e.g., on Hausdorff distance, or any standard (dis)similarity measure.)

In real applications, $I$ is defined on a finite set $\mathbb{S} \subset \mathbb{Z}^{2}$, and so is the space of (potential) solutions of Eq. (47). However, the size $\mathcal{O}\left(|\mathbb{V}|^{|\mathbb{S}|}\right)$ of this space is huge. Then, one has to accept to only look for an approximate solution of Eq. (47), instead of an exact one. In this context, a tractable strategy is to consider the homotopy-guided approach initially developped 

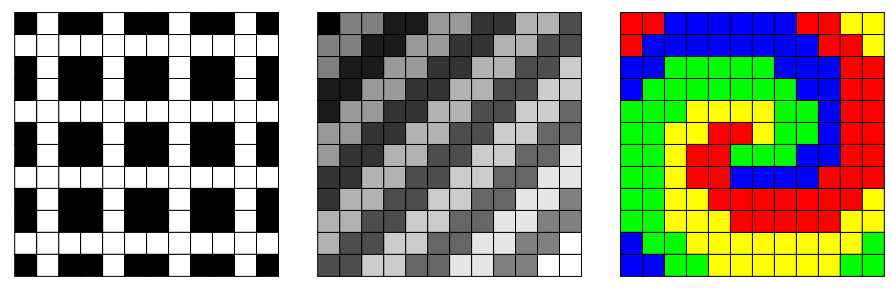

Fig. 3. Some well-composed images that cannot be regularised without a super-resolution approach, due to fine texture effects.

for monotonic transformations [41], and then adapted to nonmonotonic ones [42], [43], [6], [38].

This strategy starts from the image $I$, and iteratively eliminates forbidden configurations by modifying the value of one point $\mathbf{p} \in \mathbb{S}$ at each iteration, until stability. The choice of $\mathbf{p}$ is guided $(i)$ by the position of the forbidden configurations, (ii) by $D_{I}$, and $($ iii) by choosing $\mathbf{p}$ as a simple point. This is feasible for $\mathbb{V}=\mathbb{B}, \mathbb{G}$ or $\mathbb{L}$ since notions of simple points have been proposed in binary [44], grey-level [45] and label cases [39].

The obtained algorithm can be seen as an extension of the ones presented in [31], [46] for well-composedness recovery, to the case of regularity recovery. In particular, it presents the same strenghts and weaknesses. Indeed, in most application cases, it will converge in linear time with respect to the number of forbidden configurations, that are often sparsely distributed within images. Nevertheless, in pathological cases (e.g., in presence of fine textures, see Fig. 3), it may not converge, or even fail. To deal with this issue, we propose hereafter a second -super-resolution- regularisation strategy.

2) Super-resolution regularisation: Let $I \in \mathfrak{I m}_{\mathbb{V}}$ (with $\mathbb{V}=\mathbb{B}$ or $\mathbb{G})$ be a $(k, \bar{k})$-image. Even before the issue of regularisation, it may happen that $I$ cannot be modified into a topologically-equivalent well-composed image, when using a strategy such as the one presented above. It is then possible to oversample $I$ by explicitely representing its "interpixel" topological structure. This can be done by embedding $I$ into the Khalimsky space [18], then leading to a new image $I_{K}^{(k, \bar{k})}$ defined as

$$
\begin{array}{|lll}
I_{K}^{(8,4)}: \mathbb{Z}^{2} & \rightarrow \mathbb{V} \\
& 2 \cdot \mathbf{p} & \mapsto I(\mathbf{p}) \\
2 \cdot \mathbf{p}+(0,1) & \mapsto \bigvee^{\leqslant} I(\mathbf{p}+\{0\} \times\{0,1\}) \\
2 \cdot \mathbf{p}+(1,0) & \mapsto \bigvee^{\leqslant} I(\mathbf{p}+\{0,1\} \times\{0\}) \\
2 \cdot \mathbf{p}+(1,1) & \mapsto \bigvee^{\leqslant} I(\mathbf{p}+\{0,1\} \times\{0,1\})
\end{array}
$$

(The image $I_{K}^{(4,8)}$ is defined by substituting $\wedge$ to $\bigvee^{\text {in }}$ Eq. (48).) The proof of the following result straightforwardly derives from these definitions.

Proposition 30: Let $I \in \mathfrak{I m}_{\mathbb{V}}$ (with $\mathbb{V}=\mathbb{B}$ or $\mathbb{G}$ ). Then we have $I_{K}^{(k, \bar{k})} \in \mathfrak{W C C}_{\mathbb{V}}$. Moreover, $I_{K}^{(k, \bar{k})}$ and $I$ have the same homotopy-type, when considered as $(k, \bar{k})$-images.

From now on, we then assume that $I \in \mathfrak{W C C}_{\mathbb{V}}$ (with $\mathbb{V}=\mathbb{B}$, $\mathbb{G}$ or $\mathbb{L}$ ). It may happen that $I$ cannot be modified into a regular image when using homotopic iterative regularisation. Once again, an oversampling strategy can be alternatively proposed. This strategy no longer relies on Khalimsky space embedding, but simply on a $2 \times 2$ super-resolution approach. More precisely, from $I \in \mathfrak{W J C}_{\mathbb{V}}$, we can define a new image

$$
\begin{array}{|lll}
I_{2 \times 2}: \mathbb{Z}^{2} & \rightarrow \mathbb{V} \\
& \mathbf{p}=(x, y) & \mapsto I((\lfloor x / 2\rfloor,\lfloor y / 2\rfloor))
\end{array}
$$

The proof of the following result straightforwardly derives from this definition.

Proposition 31: Let $I \in \mathfrak{W J C}_{\mathbb{V}}$ (with $\mathbb{V}=\mathbb{B}, \mathbb{G}$ or $\mathbb{L}$ ). Then we have $I_{2 \times 2} \in \mathfrak{R e g} \mathfrak{g}_{\mathbb{V}}$. Moreover, $I_{2 \times 2}$ and $I$ have the same homotopy-type when considered as $(k, \bar{k})$ - (resp. wc-) images.

Finally, Eqs. (48)-(49) provide a global super-resolution strategy that enables to redefine any $(8,4)-,(4,8)-$, or $w c$ image as a regular -and thus topologically invariant- one. By opposition to the previous strategy, this one has the advantages of being deterministic and geometrically preserving (up to a possible "thickening" of the interpixel space). Its main drawback, by opposition to the first strategy, is its higher spatial cost, as it models an image of size $|\mathbb{S}|$ as a new one of size $4 .|\mathbb{S}|$ (and 16.|S| in the worst cases).

\section{CONCLUSION}

We have investigated the notion of topology preservation of $2 \mathrm{D}$ digital images under rigid transformation. Based on theoretical results established in the digital topology framework, we have derived efficient algorithms for analysing and preprocessing such images. The genericity of these results and methods, in terms of topological models (dual adjacency and well-composedness) and values (binary, grey-level and label images), authorise their actual use in real applications.

A short term purpose will be to prove that the notion of regularity provides not only sufficient, but also necessary conditions for topological invariance (in other words, that the $\subseteq$ symbols in Ths. 14, 17 and 22, are indeed = symbols). To this end, it will be necessary to define a relevant local topological invariant, relying, e.g., on the topological structure that can be defined on tilings of $\mathbb{Z}^{2}$ induced by rigid transformations.

We will also investigate the links between our results, established in a discrete framework, and some results obtained in the research field of digitisation, that intrinsically merges both discrete and continuous frameworks. Indeed, as suggested by Eqs. (14)-(15) the rigid transformation of a digital image can be interpreted as the (re)digitisation of its associated continuous pixel-based representation. Based on this assertion, our notion of regularity may be seen as a discrete analogue of the notion of $r$-regularity developped fifteen years ago [47], [48], for topology-preserving digitisation purpose. These links, that are easy to intuit, are less trivial to formally establish.

From a more methodological viewpoint, the next step will consist of passing from $\mathbb{Z}^{2}$ to $\mathbb{Z}^{3}$. This raises supplementary difficulties, related to the more complex definitions of topological models [49] and topological invariants [30]. To cope with this challenge, various ways may be considered. A first one relies on the possible analogy between regularity and $r$ regularity (see above). A second one relies on a morphological interpretation of regularity. Indeed, as stated in Rem. 13, regular images are open for square structuring elements, but the counterpart is not true. A specific class of open images, for which the opening relies on homotopic erosions and dilations, 
may be considered and compared to the family of regular images, in a morpho-topological framework [50], [51], [52].

\section{ACKNOWLEDGEMENT}

The research leading to these results has received funding from the French Agence Nationale de la Recherche (Grant Agreement ANR-2010-BLAN-0205).

\section{APPENDIX}

\section{A. Auxiliary properties}

The following two properties deal with configurations that have already been discussed in the literature (see, e.g., [14], [15]. Their proofs, that do not present much difficulties, are left to the reader. (We recall that we are still under the hypotheses of Rem. 8.) that

Property 32: Let $\mathbf{p} \in \mathbb{Z}^{2}$. There exists $T \in \mathfrak{R i g}_{\mathbb{Z}^{2}}$ such

$$
\mathbf{p} \notin T\left(\mathbb{Z}^{2}\right)
$$

Let $T$ be such a transformation. Let $\{\mathbf{n}, \mathbf{e}, \mathbf{s}, \mathbf{w}\}=\left\{\mathbf{q} \in \mathbb{Z}^{2} \mid\right.$ $\left.\mathbf{p} \frown_{4} \mathbf{q}\right\}$, with $\mathbf{n} \frown_{8} \mathbf{e} \frown_{8} \mathbf{S} \frown_{8} \mathbf{w}$. There exist distinct points $\mathbf{n}^{\prime}, \mathbf{e}^{\prime}, \mathbf{s}^{\prime}, \mathbf{w}^{\prime} \in \mathbb{Z}^{2}$, with $\mathbf{n}^{\prime} \frown_{4} \mathbf{e}^{\prime} \frown_{4} \mathbf{s}^{\prime} \frown_{4} \mathbf{w}^{\prime} \frown_{4} \mathbf{n}^{\prime}$, such that

$$
\forall \mathbf{q} \in\{\mathbf{n}, \mathbf{e}, \mathbf{s}, \mathbf{w}\}, \mathbf{q}=T\left(\mathbf{q}^{\prime}\right)
$$

Property 33: Let $C=\{x, x+1\} \times\{y, y+1\} \subset \mathbb{Z}^{2}$. Let $T \in \mathfrak{R i g}_{\mathbb{Z}^{2}}$. Then we have $T^{-1}(C) / \sim_{4}=\left\{T^{-1}(C)\right\}$.

\section{B. Proof of Proposition 11}

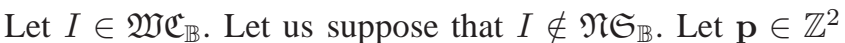
be such that $\forall \mathbf{q} \in \mathbb{Z}^{2},\left(\mathbf{q} \frown_{4} \mathbf{p}\right) \Rightarrow(I(\mathbf{p}) \neq I(\mathbf{q}))$ (Def. 10). Then, Th. 23 implies that $\forall \mathbf{q} \in \mathbb{Z}^{2},\left(\mathbf{q} \frown_{8} \mathbf{p}\right) \Rightarrow(I(\mathbf{p}) \neq$ $I(\mathbf{q})$ ), i.e., $\{\mathbf{p}\} \in \mathcal{C}^{(k, \bar{k})}[I]$. From Prop. 32 (Eq. (50)), there exists $T \in \mathfrak{R i} \mathfrak{g}_{\mathbb{Z}^{2}}$ such that $\mathbf{p} \notin T\left(\mathbb{Z}^{2}\right)$. Such a transformation $T$ does not induce a bijection between $\mathcal{C}^{(k, \bar{k})}[I]$ and $\mathcal{C}^{(k, \bar{k})}[I \circ T]$, and a fortiori an isomorphism between $\mathfrak{T}^{(k, \bar{k})}(I)$ and $\mathfrak{T}^{(k, \bar{k})}(I \circ T)$, and thus we have $I \notin \mathfrak{I n v}_{\mathbb{B}}^{(k, \bar{k})}$ (Def. 7). By

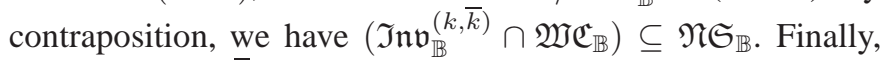
$\mathfrak{I n v}_{\mathbb{B}}^{w c} \subseteq \mathfrak{I n v}_{\mathbb{B}}^{(k, \bar{k})}$ is a straightforward consequence of Defs. 3, 7.

\section{Proof of Proposition 26}

Let $I \in \mathfrak{R e g}_{\mathbb{B}}^{k}$. Let $\mathbf{p} \in I^{-1}(\{1\})$. Since $I \in \mathfrak{N S}_{\mathbb{B}}$ (Def. 12), there exists $\mathbf{q} \in I^{-1}(\{1\})$ such that $\mathbf{p} \frown_{4} \mathbf{q}$. Then Eq. (18) forbids Eqs. (39)-(40).

Let us suppose that for all $\mathbf{p} \in I^{-1}(\{1\})$ Eqs. (39)-(40) are not verified. Let $\mathbf{p} \in I^{-1}(\{1\})$. As $\mathbf{p}$ does not verify Eq. (39), we choose $\mathbf{q} \in I^{-1}(\{1\})$ such that $\mathbf{p} \frown_{4} \mathbf{q}$. Up to $\pi / 2$ rotations, we can set $\mathbf{q}=\mathbf{p}+(0,1)$. Since $\mathbf{p}$ does not verify Eq. (39), we have $\mathbf{p}+(1,0)$ or $\mathbf{p}-(1,0) \in I^{-1}(\{1\})$. Up to symmetries, we can set $\mathbf{p}+(1,0) \in I^{-1}(\{1\})$. If $\mathbf{p}+(1,1) \in$ $I^{-1}(\{1\})$, then, $\mathbf{p}, \mathbf{q}$ satisfy the RHS of Eq. (18). Let us now suppose that $\mathbf{p}+(1,1) \in I^{-1}(\{0\})$. Since $\mathbf{q}$ does not verify Eq. (39), we have $\mathbf{p}+(-1,1) \in I^{-1}(\{1\})$. But Eq. (40) implies $\mathbf{p}-(1,0) \in I^{-1}(\{1\})$, and $\mathbf{p}, \mathbf{q}$ then satisfy the RHS of Eq. (18). Then, $I \in \mathfrak{R e} \mathfrak{g}_{\mathbb{B}}^{k}$.

The result follows by contraposition. The same reasonning holds for $\mathfrak{R e} \mathfrak{g}_{\mathbb{B}}^{\bar{k}}$.

\section{Proof of Theorem 14}

Proposition 34: Let $I \in \mathfrak{R e} \mathfrak{g}_{\mathbb{B}}^{k}$ (resp. $\mathfrak{R e} \mathfrak{g}_{\mathbb{B}}^{\bar{k}}$, resp. $\mathfrak{R e} \mathfrak{g}_{\mathbb{B}}$ ). Let $T \in \mathfrak{R i g}_{\mathbb{Z}^{2}}$. Then $T_{\mid(I \circ T)^{-1}(\{1\})}$ establishes a homomorphism from $\left((I \circ T)^{-1}(\{1\}), \frown_{4}\right)\left(\operatorname{resp} .\left((I \circ T)^{-1}(\{1\}), \frown_{8}\right.\right.$ ), resp. $\left.\left((I \circ T)^{-1}(\{1\}), \frown_{4}\right)\right)$ to $\left(I^{-1}(\{1\}), \sim_{4}\right)$ (resp. $\left(I^{-1}(\{1\}), \sim_{8}\right)$, resp. $\left.\left(I^{-1}(\{1\}), \sim_{4}\right)\right)$, while $T_{\mid(I \circ T)^{-1}(\{0\})}$ establishes a homomorphism from $\left((I \circ T)^{-1}(\{0\}), \frown_{8}\right)$ (resp. $\left((I \circ T)^{-1}(\{0\}), \frown_{4}\right)$, resp. $\left.\left((I \circ T)^{-1}(\{0\}), \frown_{4}\right)\right)$ to $\left(I^{-1}(\{0\}), \sim_{8}\right)$ (resp. $\left(I^{-1}(\{0\}), \sim_{4}\right)$, resp. $\left(I^{-1}(\{0\}), \sim_{4}\right)$ ).

Proof Let $I \in \mathfrak{R e} \mathfrak{g}_{\mathbb{B}}$. Let $\mathbf{p}^{\prime}, \mathbf{q}^{\prime} \in(I \circ T)^{-1}(\{1\})$, with $\mathbf{p}^{\prime} \frown_{4} \mathbf{q}^{\prime}$. Let $\mathbf{p}=T\left(\mathbf{p}^{\prime}\right), \mathbf{q}=T\left(\mathbf{q}^{\prime}\right)$. From Eq. (13), two cases can occur: $(i) \mathbf{p}=\mathbf{q}$, and then $\mathbf{p} \sim_{4} \mathbf{q}$; $(i i) \mathbf{p} \frown_{8} \mathbf{q}$, that implies $\mathbf{p} \sim_{4} \mathbf{q}$ (Def. 3), and then $\mathbf{p} \sim_{4} \mathbf{q}$. The same reasonning holds for $I \in \mathfrak{R e} \mathfrak{g}_{\mathbb{B}}$ and $(I \circ T)^{-1}(\{0\}) ; I \in \mathfrak{R e} \mathfrak{g}_{\mathbb{B}}^{k}$ and $(I \circ T)^{-1}(\{1\})$; and $I \in \mathfrak{R e} \mathfrak{g}_{\mathbb{B}}^{\bar{k}}$ and $(I \circ T)^{-1}(\{0\})$.

Let $I \in \mathfrak{R e} \mathfrak{g}_{\mathbb{B}}^{k}$. Let $\mathbf{p}^{\prime}, \mathbf{q}^{\prime} \in(I \circ T)^{-1}(\{0\})$, with $\mathbf{p}^{\prime} \frown_{8} \mathbf{q}^{\prime}$. Let $\mathbf{p}=T\left(\mathbf{p}^{\prime}\right), \mathbf{q}=T\left(\mathbf{q}^{\prime}\right)$. From Eq. (13), three cases can occur: $(i) \mathbf{p}=\mathbf{q}$, and then $\mathbf{p} \sim_{8} \mathbf{q}$; $(i i) \mathbf{p} \frown_{8} \mathbf{q}$, and then $\mathbf{p} \sim_{8} \mathbf{q}$; or $($ iii $) \mathbf{p}=\mathbf{q}+(2,0)$ or $(2,1)$, up to $\pi / 2$ rotations and symmetries, and then $\mathbf{p} \sim_{8} \mathbf{q}$ derives from Prop. 26. The same reasonning holds for $I \in \mathfrak{R e} \mathfrak{g}_{\mathbb{B}}^{\bar{k}}$ and $(I \circ T)^{-1}(\{1\})$.

We can then licitely define the following notions. Let $I \in$

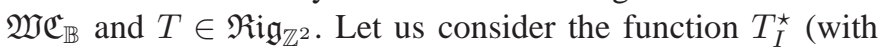
$\star=(k, \bar{k})$ or $w c)$ defined as

$$
\begin{array}{|lll}
T_{I}^{\star}: \mathcal{C}^{\star}[I \circ T] & \rightarrow \mathcal{C}^{\star}[I] \\
& C & \mapsto C_{T} \supseteq T(C)
\end{array}
$$

We are now ready to establish the first part of the isomorphism, namely the one-to-one correspondence between the connected components of the initial and transformed images.

Proposition 35: Let $I \in \mathfrak{R e g} \mathfrak{g}_{\mathbb{B}}^{k}$ (resp. $\mathfrak{R e} \mathfrak{g}_{\mathbb{B}}^{\bar{k}}$, resp. $\mathfrak{R e g} \mathfrak{g}_{\mathbb{B}}$ ).

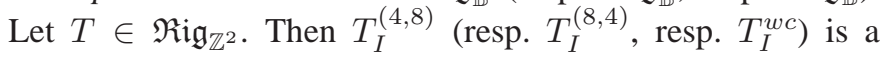
bijection.

Proof Let $C \in \mathcal{C}^{\star}[I]$ and $\mathbf{p} \in C$. Since $I \notin \mathfrak{N S}_{\mathbb{B}}$, we can choose $\mathbf{q} \in C$ such that $\mathbf{p} \frown_{4} \mathbf{q}$. Then, from Prop. 32 (Eq. (50)), there exists $\mathbf{p}^{\prime} \in \mathbb{Z}^{2}$ such that $T\left(\mathbf{p}^{\prime}\right) \in\{\mathbf{p}, \mathbf{q}\} \subseteq C$. Thus, $T_{I}^{\star}$ is a surjection.

We assume that $I \in \mathfrak{R e} \mathfrak{g}_{\mathbb{B}}$. Let $\mathbf{p}^{\prime}, \mathbf{q}^{\prime} \in(I \circ T)^{-1}(\{1\})$. Let $\mathbf{p}=T\left(\mathbf{p}^{\prime}\right), \mathbf{q}=T\left(\mathbf{q}^{\prime}\right)$. Let us suppose that $\mathbf{p}, \mathbf{q} \in C \in$ $I^{-1}(\{1\}) / \sim_{4}$. We have $\mathbf{p} \sim_{4} \mathbf{q}$, i.e., there exists a set $\left\{\mathbf{p}_{i}\right\}_{i=0}^{k}$ $(k \geqslant 0)$ such that $\mathbf{p}_{0}=\mathbf{p}, \mathbf{p}_{k}=\mathbf{q}, \mathbf{p}_{i} \in I^{-1}(\{1\})$ for any $i \in$ $\llbracket 0, k \rrbracket$, and $\mathbf{p}_{i} \frown_{4} \mathbf{p}_{i+1}$ for any $i \in \llbracket 0, k-1 \rrbracket$. Let $i \in \llbracket 0, k-1 \rrbracket$. If $\mathbf{p}_{i}, \mathbf{p}_{i+1} \in T\left(\mathbb{Z}^{2}\right)$, we set $\mathbf{p}_{i}^{\prime}, \mathbf{p}_{i+1}^{\prime} \in(I \circ T)^{-1}(\{1\})$ such that $T\left(\mathbf{p}_{i}^{\prime}\right)=\mathbf{p}_{i}$ and $T\left(\mathbf{p}_{i+1}^{\prime}\right)=\mathbf{p}_{i+1}$; it then derives from Prop. 33 and Def. 12 that $\mathbf{p}_{i}^{\prime} \sim_{4} \mathbf{p}_{i+1}^{\prime}$. Let us now suppose that $\mathbf{p}_{i}$ or $\mathbf{p}_{i+1} \notin T\left(\mathbb{Z}^{2}\right)$, for instance $\mathbf{p}_{i+1} \notin T\left(\mathbb{Z}^{2}\right)$. It derives from Prop. 32 (Eq. (51)) that $\mathbf{p}_{i+2} \in T\left(\mathbb{Z}^{2}\right)$ (for the same reasons, we cannot have $\mathbf{p}_{i}, \mathbf{p}_{i+1} \notin T\left(\mathbb{Z}^{2}\right)$ ). We set $\mathbf{p}_{i}^{\prime}, \mathbf{p}_{i+2}^{\prime} \in$ $(I \circ T)^{-1}(\{1\})$ such that $T\left(\mathbf{p}_{i}^{\prime}\right)=\mathbf{p}_{i}$ and $T\left(\mathbf{p}_{i+2}^{\prime}\right)=\mathbf{p}_{i+2}$. From Prop. 32 (Eq. (51)), we then have $\mathbf{p}_{i}^{\prime} \sim_{4} \mathbf{p}_{i+2}^{\prime}$. Then $\Pi=T^{-1}\left(\left\{p_{i}\right\}_{i=0}^{k}\right)$ is such that $\mathbf{p}^{\prime}, \mathbf{q}^{\prime} \in \Pi \subseteq(I \circ T)^{-1}(\{1\})$ and that there exists $C_{T} \in(I \circ T)^{-1}(\{1\}) / \sim_{4}$ such that $\Pi \subseteq$ $C_{T}$. The same reasonning holds for $\mathfrak{R e} \mathfrak{g}_{\mathbb{B}}$ and $(I \circ T)^{-1}(\{0\})$; $\mathfrak{R e} \mathfrak{g}_{\mathbb{B}}^{k}$ and $(I \circ T)^{-1}(\{1\})$; and $\mathfrak{R e} \mathfrak{g}_{\mathbb{B}}^{\bar{k}}$ and $(I \circ T)^{-1}(\{0\})$.

We now assume that $I \in \mathfrak{R e} \mathfrak{g}_{\mathbb{B}}^{k}$. Let $\mathbf{p}^{\prime}, \mathbf{q}^{\prime} \in(I \circ T)^{-1}(\{0\})$. Let $\mathbf{p}=T\left(\mathbf{p}^{\prime}\right), \mathbf{q}=T\left(\mathbf{q}^{\prime}\right)$. Let us suppose that $\mathbf{p}, \mathbf{q} \in$ $C \in I^{-1}(\{0\}) / \sim_{8}$. As $I \in \mathfrak{W C}_{\mathbb{B}}$, we actually have $C \in$ 
$I^{-1}(\{0\}) / \sim_{4}$. Then, we have $\mathbf{p} \sim_{4} \mathbf{q}$, i.e., there exists a set $\left\{\mathbf{p}_{i}\right\}_{i=0}^{k}(k \geqslant 0)$ such that $\mathbf{p}_{0}=\mathbf{p}, \mathbf{p}_{k}=\mathbf{q}, \mathbf{p}_{i} \in I^{-1}(\{0\})$ for any $i \in \llbracket 0, k \rrbracket$, and $\mathbf{p}_{i} \frown \sim_{4} \mathbf{p}_{i+1}$ for any $i \in \llbracket 0, k-1 \rrbracket$. Let $i \in \llbracket 0, k-1 \rrbracket$. If $\mathbf{p}_{i}, \mathbf{p}_{i+1} \in T\left(\mathbb{Z}^{2}\right)$, we set $\mathbf{p}_{i}^{\prime}, \mathbf{p}_{i+1}^{\prime}$ such that $T\left(\mathbf{p}_{i}^{\prime}\right)=\mathbf{p}_{i}$ and $T\left(\mathbf{p}_{i+1}^{\prime}\right)=\mathbf{p}_{i+1}$; it then derives from Eq. (13) that $\mathbf{p}_{i}^{\prime} \sim_{8} \mathbf{p}_{i+1}^{\prime}$. Let us now suppose that $\mathbf{p}_{i}$ or $\mathbf{p}_{i+1} \notin T\left(\mathbb{Z}^{2}\right)$, for instance $\mathbf{p}_{i+1} \notin T\left(\mathbb{Z}^{2}\right)$. It derives from Prop. 32 (Eq. (51)) that $\mathbf{p}_{i+2} \in T\left(\mathbb{Z}^{2}\right)$ (for the same reasons, we cannot have $\left.\mathbf{p}_{i}, \mathbf{p}_{i+1} \notin T\left(\mathbb{Z}^{2}\right)\right)$. We set $\mathbf{p}_{i}^{\prime}, \mathbf{p}_{i+2}^{\prime}$ such that $T\left(\mathbf{p}_{i}^{\prime}\right)=\mathbf{p}_{i}$ and $T\left(\mathbf{p}_{i+2}^{\prime}\right)=\mathbf{p}_{i+2}$. From Prop. 32 (Eq. (51)), we then have $\mathbf{p}_{i}^{\prime} \sim_{4} \mathbf{p}_{i+2}^{\prime}$. Then $\Pi=T^{-1}\left(\left\{p_{i}\right\}_{i=0}^{k}\right)$ is such that $\mathbf{p}^{\prime}, \mathbf{q}^{\prime} \in \Pi \subseteq(I \circ T)^{-1}(\{0\})$ and that there exists $C_{T} \in(I \circ T)^{-1}(\{0\}) / \sim_{8}$ such that $\Pi \subseteq C_{T}$. The same reasonning holds for $\mathfrak{R e g}_{\mathbb{B}}^{\bar{k}}$ and $(I \circ T)^{-1}(\{1\})$.

It straightforwardly follows from these two sub-reasonnings that $T_{I}^{\star}$ is indeed an injection.

The following proposition is a consequence of this result.

Proposition 36: Let $I \in \mathfrak{R e g}_{\mathbb{B}}^{k}$ (resp. $\mathfrak{R e} \mathfrak{g}_{\mathbb{B}}^{\bar{k}}$, resp. $\mathfrak{R e g} \mathfrak{g}_{\mathbb{B}}$ ). Let $T \in \mathfrak{R i g}_{\mathbb{Z}^{2}}$. We have $I \circ T \in \mathfrak{I m}_{\mathbb{B}}$ (resp. $\mathfrak{I m}_{\mathbb{B}}$, resp. $\left.\mathfrak{W C}_{\mathbb{B}}\right)$. Moreover, we have $T_{I}^{\star}\left(B_{I \circ T}^{\star}\right)=B_{I}^{\star}$.

Proof The fact that $I \circ T \in \mathfrak{I m}_{\mathbb{B}}$ straightforwardly derives from the fact that $T_{I}^{\star}$ is a bijection, and from the definition of $T$ (Eq. (13)), that implies that for any $\mathbf{p} \in \mathbb{Z}^{2}, T^{-1}(\{\mathbf{p}\})$ is finite. We have $T_{I}^{\star}\left(B_{I \circ T}^{\star}\right)=B_{I}^{\star}$ for the very same reasons. Let us now consider that $I \circ T \notin \mathfrak{W J C}_{\mathbb{B}}$. Then, from Th. 23, there exist distinct points $\mathbf{n}, \mathbf{e}, \mathbf{s}, \mathbf{w} \in \mathbb{Z}^{2}$, with $\mathbf{n} \frown_{4} \mathbf{e} \frown_{4}$ $\mathbf{s} \frown_{4} \mathbf{w} \frown_{4} \mathbf{n}$, that verify Eq. (36). From Eq. (13), we then derive that there exist distinct points $\mathbf{n}^{\prime}, \mathbf{e}^{\prime}, \mathbf{s}^{\prime}, \mathbf{w}^{\prime} \in \mathbb{Z}^{2}$ such that $T(\mathbf{n})=\mathbf{n}^{\prime}, T(\mathbf{e})=\mathbf{e}^{\prime}, T(\mathbf{s})=\mathbf{s}^{\prime}, T(\mathbf{w})=\mathbf{w}^{\prime}$. Still from Eq. (13), we have moreover $\mathbf{n}^{\prime} \frown_{8} \mathbf{e}^{\prime} \frown_{8} \mathbf{s}^{\prime} \frown_{8} \mathbf{w}^{\prime} \frown_{8}$ $\mathbf{n}^{\prime}$. These equalities authorise only three configurations, up to $\pi / 2$ rotations and symmetries: $(i) \mathbf{e}^{\prime}=\mathbf{n}^{\prime}+(1,0), \mathbf{s}^{\prime}=$ $\mathbf{n}^{\prime}+(2,-1), \mathbf{w}^{\prime}=\mathbf{n}^{\prime}+(1,-1) ;(i i) \mathbf{e}^{\prime}=\mathbf{n}^{\prime}+(1,0), \mathbf{s}^{\prime}=$ $\mathbf{n}^{\prime}+(1,-1), \mathbf{w}^{\prime}=\mathbf{n}^{\prime}+(0,-1) ;($ iii $) \mathbf{e}^{\prime}=\mathbf{n}^{\prime}+(1,-1), \mathbf{s}^{\prime}=$ $\mathbf{n}^{\prime}-(2,0), \mathbf{w}^{\prime}=\mathbf{n}^{\prime}-(1,1)$. Configuration $(i)$ corresponds to Eq. (40), configuration (ii) corresponds to Eq. (36), and configuration (iii) corresponds to Eq. (39); in each case, we have $I \notin \mathfrak{R e} \mathfrak{g}_{\mathbb{B}}$. By contrapotision, $I \notin \mathfrak{R e} \mathfrak{g}_{\mathbb{B}}$ implies that $I \circ T \in \mathfrak{W C C}_{\mathbb{B}}$.

We are now ready to establish the last part of the isomorphism, namely the preservation of the adjacency relation.

Proposition 37: Let $I \in \mathfrak{R e g}_{\mathbb{B}}^{k}$ (resp. $\mathfrak{R e} \mathfrak{g}_{\mathbb{B}}^{\bar{k}}$, resp. $\mathfrak{R e g} \mathfrak{g}_{\mathbb{B}}$ ). Let $T \in \mathfrak{R i g}_{\mathbb{Z}^{2}}$. Let $C_{1}, C_{2} \in \mathcal{C}^{\star}[I \circ T]$ with $\star=(4,8)$ (resp. $(8,4)$, resp. $w c)$. We have

$$
\left(C_{1} \frown_{I \circ T}^{\star} C_{2}\right) \Longleftrightarrow\left(T_{I}^{\star}\left(C_{1}\right) \frown_{I}^{\star} T_{I}^{\star}\left(C_{2}\right)\right)
$$

Proof Let $C_{1} \frown_{I \circ T}^{\star} C_{2}$. Up to reindexing, we have $C_{1} \subseteq$ $(I \circ T)^{-1}(\{0\})$ and $C_{2} \subseteq(I \circ T)^{-1}(\{1\})$. Let $\mathbf{p}^{\prime} \in C_{1}$, $\mathbf{q}^{\prime} \in C_{2}$ be such that $\mathbf{p}^{\prime} \frown_{4} \mathbf{q}^{\prime}$. Let $\mathbf{p}=T\left(\mathbf{p}^{\prime}\right) \in T_{I}^{\star}\left(C_{1}\right)$, $\mathbf{q}=T\left(\mathbf{q}^{\prime}\right) \in T_{I}^{\star}\left(C_{1}\right)$. From Eq. (13), we can have: $(i) \mathbf{p} \frown_{4} \mathbf{q}$ and then $T_{I}^{\star}\left(C_{1}\right) \frown_{I}^{\star} T_{I}^{\star}\left(C_{2}\right)$; or $(i i) \mathbf{p} \frown_{8} \mathbf{q}$ and $\mathbf{p} \Varangle_{4} \mathbf{q}$. In that second case, let $\mathbf{r} \in \mathbb{Z}^{2}$ be such that $\mathbf{p} \frown_{4} \mathbf{r} \frown_{4} \mathbf{q}$. We have either $\mathbf{r} \in T_{I}^{\star}\left(C_{1}\right)$ or $T_{I}^{\star}\left(C_{2}\right)$, and then $T_{I}^{\star}\left(C_{1}\right) \frown_{I}^{\star}$ $T_{I}^{\star}\left(C_{2}\right)$.

Let $T_{I}^{\star}\left(C_{1}\right) \frown_{I}^{\star} T_{I}^{\star}\left(C_{2}\right)$. Up to reindexing, we have $T_{I}^{\star}\left(C_{1}\right) \subseteq T^{-1}(\{0\})$ and $T_{I}^{\star}\left(C_{2}\right) \subseteq T^{-1}(\{1\})$. Let $\mathbf{p} \in$ $T_{I}^{\star}\left(C_{1}\right), \mathbf{q} \in T_{I}^{\star}\left(C_{2}\right)$ be such that $\mathbf{p} \frown_{4} \mathbf{q}$. Case 1: there exist $\mathbf{p}^{\prime} \in C_{1}$ such that $T\left(\mathbf{p}^{\prime}\right)=\mathbf{p}$, and $\mathbf{q}^{\prime} \in C_{2}$ such that $T\left(\mathbf{q}^{\prime}\right)=\mathbf{q}$. From Eq. (13), three possibilities can occur: $(i)$ $\mathbf{p}^{\prime} \frown_{4} \mathbf{q}^{\prime}$ and then $C_{1} \frown_{I}^{\star} C_{2} ;(i i) \mathbf{p}^{\prime} \frown_{8} \mathbf{q}^{\prime}$ and $\mathbf{p}^{\prime} \succ_{4} \mathbf{q}^{\prime}$, and by choosing $\mathbf{r}^{\prime} \in \mathbb{Z}^{2}$ such that $\mathbf{p}^{\prime} \frown_{4} \mathbf{r}^{\prime} \frown_{4} \mathbf{q}^{\prime}$, we have either $\mathbf{r}^{\prime} \in C_{1}$ or $C_{2}$, and then $C_{1} \frown_{I}^{\star} C_{2} ;($ iii $) \mathbf{q}^{\prime}=\mathbf{p}^{\prime}+(2,0)$, up to $\pi / 2$ rotations, and by choosing $\mathbf{r}^{\prime}=\mathbf{p}^{\prime}+(1,0)$, we have either $\mathbf{r}^{\prime} \in C_{1}$ or $C_{2}$, and then $C_{1} \frown_{I}^{\star} C_{2}$. Case $2: \mathbf{p} \notin T\left(\mathbb{Z}^{2}\right)$

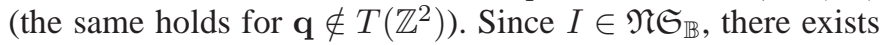
$\mathbf{r} \in T_{I}^{\star}\left(C_{1}\right)$ and $\mathbf{s} \in T_{I}^{\star}\left(C_{2}\right)$ such that $\mathbf{p} \frown_{4} \mathbf{r} \frown_{8} \mathbf{s} \frown_{4} \mathbf{p}$. Then, from Prop. 32 (Eq. (51)), there exists $\mathbf{r}^{\prime} \in C_{1}$ such that $T\left(\mathbf{r}^{\prime}\right)=\mathbf{r}$, and $\mathbf{s}^{\prime} \in C_{2}$ such that $T\left(\mathbf{s}^{\prime}\right)=\mathbf{s}$, and $\mathbf{r}^{\prime} \frown_{4} \mathbf{s}^{\prime}$, and then $C_{1} \frown_{I}^{\star} C_{2}$.

By gathering Props. 35-37, we obtain Th. 14.

\section{REFERENCES}

[1] D. L. Pham, P.-L. Bazin, and J. L. Prince, "Digital topology in brain imaging,” IEEE Signal Proc. Mag., vol. 27, no. 4, pp. 51-59, 2010.

[2] P. Soille and M. Pesaresi, "Advances in mathematical morphology applied to geoscience and remote sensing," IEEE T. Geosci. Remote, vol. 40, no. 9, pp. 2042-2055, 2002.

[3] A. Rosenfeld and J. L. Pfaltz, "Sequential operations in digital picture processing," J. ACM, vol. 13, no. 4, pp. 471-494, 1966.

[4] O. Duda, P. E. Hart, and J. H. Munson, "Graphical data processing research study and experimental investigation," Stanford Research Institute, Tech. Rep. AD650926, 1967.

[5] P.-L. Bazin, L. M. Ellingsen, and D. L. Pham, "Digital homeomorphisms in deformable registration," in IPMI, Proc., ser. LNCS, vol. 4584. Springer, 2007, pp. 211-222.

[6] S. Faisan, N. Passat, V. Noblet, R. Chabrier, and C. Meyer, "Topologypreserving warping of binary images according to one-to-one mappings," IEEE T. Image Process., vol. 20, no. 8, pp. 2135-2145, 2011.

[7] V. Noblet, C. Heinrich, F. Heitz, and J.-P. Armspach, "3-D deformable image registration: A topology preservation scheme based on hierarchical deformation models and interval analysis optimization," IEEE T. Image Process., vol. 14, no. 5, pp. 553-566, 2005.

[8] P. Ngo, Y. Kenmochi, N. Passat, and H. Talbot, "Combinatorial structure for rigid transformations in 2D digital images," Comput. Vis. Image Und., In Press, DOI: 10.1016/j.cviu.2012.08.014.

[9] E. Andres, "The quasi-shear rotation," in DGCI, Proc., ser. LNCS, vol. 1176. Springer, 1996, pp. 307-314.

[10] J.-P. Reveillès, "Géométrie discrète, calcul en nombres entiers et algorithmique," Thèse d'État, Université Strasbourg 1, 1991.

[11] M. S. Richman, "Understanding discrete rotations," in IEEE ICASSP, Proc., vol. 3, 1997, pp. 2057-2060.

[12] B. Nouvel and E. Rémila, "Incremental and transitive discrete rotations," in IWCIA, Proc., ser. LNCS, vol. 4040. Springer, 2006, pp. 199-213.

[13] Y. Thibault, Y. Kenmochi, and A. Sugimoto, "Computing upper and lower bounds of rotation angles from digital images," Pattern Recogn., vol. 42, no. 8, pp. 1708-1717, 2009.

[14] B. Nouvel and E. Rémila, "Configurations induced by discrete rotations: Periodicity and quasi-periodicity properties," Discrete Appl. Math., vol. 147, no. 2-3, pp. 325-343, 2005.

[15] P. Ngo, Y. Kenmochi, N. Passat, and H. Talbot, "Sufficient conditions for topological invariance of 2D digital images under rigid transformations," in DGCI, Proc., ser. LNCS, vol. 7749. Springer., 2013, pp. 155-168.

[16] T. Y. Kong and A. Rosenfeld, "Digital topology: Introduction and survey," Comput. Vision Graph., vol. 48, no. 3, pp. 357-393, 1989.

[17] L. Mazo, N. Passat, M. Couprie, and C. Ronse, "Digital imaging: A unified topological framework," J. Math. Imaging Vis., vol. 44, no. 1, pp. 19-37, 2012.

[18] E. Khalimsky, "Topological structures in computer science," J. Appl. Math. Sim., vol. 1, no. 1, pp. 25-40, 1987.

[19] V. A. Kovalevsky, "Finite topology as applied to image analysis," Comput. Vision Graph., vol. 46, no. 2, pp. 141-161, 1989.

[20] L. Mazo, N. Passat, M. Couprie, and C. Ronse, "Paths, homotopy and reduction in digital images," Acta Appl. Math., vol. 113, no. 2, pp. 167193, 2011.

[21] C. R. F. Maunder, Algebraic Topology. Dover, New York, 1996.

[22] A. Rosenfeld, "Digital topology," Am. Math. Mon., vol. 86, no. 8, pp. 621-630, 1979.

[23] L. J. Latecki, U. Eckhardt, and A. Rosenfeld, "Well-composed sets," Comput. Vis. Image Und., vol. 61, no. 1, pp. 70-83, 1995. 
[24] T. Y. Kong, “A digital fundamental group," Comput. Graph., vol. 13, no. 2, pp. 159-166, 1989.

[25] C. Ronse, "A topological characterization of thinning," Theor. Comput. Sci., vol. 43, no. 1, pp. 31-41, 2007.

[26] G. Bertrand and G. Malandain, "A new characterization of threedimensional simple points," Pattern Recogn. Lett., vol. 15, no. 2, pp. $169-175,1994$.

[27] G. Bertrand, "On P-simple points," C.R. Acad. Sci. I-Math., vol. I, no. 321, pp. 1077-1084, 1995.

[28] N. Passat and L. Mazo, "An introduction to simple sets," Pattern Recogn. Lett., vol. 30, no. 15, pp. 1366-1377, 2009.

[29] A. Rosenfeld, "Adjacency in digital pictures," Inform. Control, vol. 26, no. 1 , pp. 24-33, 1974

[30] G. Bertrand, M. Couprie, and N. Passat, "A note on 3-D simple points and simple-equivalence," Inform. Process. Lett., vol. 109, no. 13, pp. 700-704, 2009.

[31] A. Rosenfeld, T. Y. Kong, and A. Nakamura, "Topology-preserving deformations of two-valued digital pictures," Graph. Model. Im. Proc., vol. 60 , no. 1 , pp. $24-34,1998$

[32] L. Najman and H. Talbot, Eds., Mathematical Morphology: From Theory to Applications. ISTE/J. Wiley \& Sons, 2010.

[33] J. Marchadier, D. Arquès, and S. Michelin, "Thinning grayscale wellcomposed images," Pattern Recogn. Lett., vol. 25, no. 5, pp. 581-590, 2004.

[34] P. Salembier, A. Oliveras, and L. Garrido, "Anti-extensive connected operators for image and sequence processing," IEEE T. Image Process., vol. 7, no. 4, pp. 555-570, 1998.

[35] P. Salembier and M. H. F. Wilkinson, "Connected operators: A review of region-based morphological image processing techniques," IEEE Signal Proc. Mag., vol. 26, no. 6, pp. 136-157, 2009.

[36] L. J. Latecki, "Multicolor well-composed pictures," Pattern Recogn. Lett., vol. 16, no. 4, pp. 425-431, 1995.

[37] Y. Cointepas, I. Bloch, and L. Garnero, "A cellular model for multiobjects multi-dimensional homotopic deformations," Pattern Recogn., vol. 34, no. 9, pp. 1785-1798, 2001.

[38] G. Damiand, A. Dupas, and J.-O. Lachaud, "Fully deformable 3D digital partition model with topological control," Pattern Recogn. Lett., vol. 32, no. 9, pp. 1374-1383, 2011.

[39] L. Mazo, N. Passat, M. Couprie, and C. Ronse, "Topology on digital label images," J. Math. Imaging Vis., vol. 44, no. 3, pp. 254-281, 2012.

[40] L. Mazo, "A framework for label images," in CTIC, Proc., ser. LNCS, vol. 7309. Springer, 2012, pp. 1-12.

[41] E. R. Davies and A. P. Plummer, "Thinning algorithms: A critique and a new methodology," Pattern Recogn., vol. 14, no. 16, pp. 53-63, 1981.

[42] J.-F. Mangin, V. Frouin, I. Bloch, J. Régis, and J. López-Krahe, "From 3D magnetic resonance images to structural representations of the cortex topography using topology preserving deformations," J. Math. Imaging Vis., vol. 5, no. 4, pp. 297-318, 1995.

[43] X. Han, C. Xu, and J. L. Prince, "A topology preserving level set method for geometric deformable models," IEEE T. Pattern Anal., vol. 25, no. 6, pp. 755-768, 2003.

[44] M. Couprie and G. Bertrand, "New characterizations of simple points in 2D, 3D, and 4D discrete spaces," IEEE T. Pattern Anal., vol. 31, no. 4, pp. 637-648, 2009.

[45] M. Couprie, F. N. Bezerra, and G. Bertrand, "Topological operators for grayscale image processing," J. Electron. Imaging, vol. 10, no. 4, pp. 1003-1015, 2001

[46] M. Siqueira, L. J. Latecki, N. J. Tustison, J. H. Gallier, and J. C. Gee, "Topological repairing of 3D digital images," J. Math. Imaging Vis., vol. 30, no. 3, pp. 249-274, 2008.

[47] A. Gross and L. Latecki, "Digitizations preserving topological and differential geometric properties," Comput. Vis. Image Und., vol. 62, no. 3, pp. 370-381, 1995 .

[48] L. J. Latecki, C. Conrad, and A. Gross, "Preserving topology by a digitization process," J. Math. Imaging Vis., vol. 8, no. 2, pp. 131-159, 1998.

[49] L. J. Latecki, "3D well-composed pictures," Comput. Vision Graph., vol. 59, no. 3, pp. 164-172, 1997.

[50] C. Ronse, "Set-theoretical algebraic approaches to connectivity in continuous or digital spaces," J. Math. Imaging Vis., vol. 8, no. 1, pp. 41-58, 1998 .

[51] J. Serra, "Connectivity on complete lattices," J. Math. Imaging Vis., vol. 9, no. 3, pp. 231-251, 1998.

[52] U. Braga-Neto and J. K. Goutsias, "A theoretical tour of connectivity in image processing and analysis," J. Math. Imaging Vis., vol. 19, no. 1, pp. 5-31, 2003. 